Article

\title{
The Influence of Flow Rates on Pressure Fluctuation in the Pump Mode of Pump-Turbine with Splitter Blades
}

\author{
Ping Huang, Yajing Xiao *, Jinfeng Zhang *, Haikun Cai and Haiqin Song \\ National Research Center of Pumps, Jiangsu University, Zhenjiang 212013, China; huangping@ujs.edu.cn (P.H.); \\ 2221711003@stmail.ujs.edu.cn (H.C.); 2221811034@stmail.ujs.edu.cn (H.S.) \\ * Correspondence: 2221911024@stmail.ujs.edu.cn (Y.X.); zhangjinfeng@ujs.edu.cn (J.Z); \\ Tel.: +86-187-9602-9957 (Y.X.); +86-137-7647-6981 (J.Z.)
}

Received: 1 September 2020; Accepted: 24 September 2020; Published: 26 September 2020

\begin{abstract}
This paper takes a pump-turbine as the research subject and, based on the Computational Fluid Dynamics (CFD) numerical method and combined with test data, investigates the pressure fluctuation characteristics in the pump mode and analyzes the pressure fluctuation characteristics at $0.75 \mathrm{Q}_{\mathrm{d}}, 1.0 \mathrm{Q}_{\mathrm{d}}$ and $1.25 \mathrm{Q}_{\mathrm{d}}$ when the guide vane opening is $17.5^{\circ}$. The results showed that the protruding frequencies of pressure fluctuation in the bladeless region were mainly $5 f_{n}, 10 f_{n}$ and $20 \mathrm{f}_{\mathrm{n}}$, and the main frequencies in the runner area and near the outlet wall of the draft tube were $16 f_{n}$ and $5 f_{n}$, respectively. At different heights for the guide vanes, the pressure fluctuation in the bladeless region had significant differences, and the pressure fluctuation near the bottom ring was the most intense. The amplitude of the rotor-stator interaction frequency continuously attenuates from the bladeless region to the outlet of the stay vanes, and the amplitude attenuation of each frequency is mainly concentrated in the area of the guide vanes. In this paper, the influence of different flow rates on the pressure fluctuation in the pump mode is analyzed, which provides a theoretical reference for the stability and further study of pump-turbines.
\end{abstract}

Keywords: pump-turbine; pump mode; flow rates; pressure fluctuation; flow characteristics

\section{Introduction}

The pumped storage power station plays a few important roles in the power system, such as peak load regulating, emergency standby and directional service. The pump-turbine, as a reversible unit, needs to take two different working conditions into account: the pump model and the turbine model. There are many overflow components, and transition conditions are frequent, so its internal flow is complex with strong pressure fluctuations. It is very important to reduce the influence of pressure fluctuations, which can induce the unit to produce vibrations of a specific frequency and lead to the resonance of components, or even vibration of the plant due to improper design. A runner with splitter blades has gradually been recognized to play an important role in reducing pressure fluctuations and improving cavitation performance. At present, foreign and domestic scholars, using computational fluid dynamics and the fluid-structure interaction numerical calculation method for analysis of the internal flow of the pump-turbine, have sufficient cognition about its mechanism and also give relevant improvement measures [1-14].

Botero [15] adopted a non-invasive measurement method to monitor and track the rotation stall of the pump-turbine and identified the frequency characteristics, and characteristics of the rotor-stator interaction under the rotation stall, which are expected to be applied to state monitoring in the actual operation of the full-size pump-turbine. Stuermer [16] focused on the impact on aerodynamic 
performance and aeroacoustics caused by the presence of a pylon and concluded that the use of active flow control, in the form of pylon trailing edge blowing, can alleviate these adverse installation effects to a notable extent. Akkermans [17] performed an aeroacoustic analysis with the Ffowcs Williams-Hawkings tool APSIM+ for both configurations and came to similar conclusions. Stuermer and Akkermans [18] presented analysis and validation of the Deutsches Zentrum für Luft- und Raumfahrt (DLR) numerical approach to coupled CFD -CAAsimulations to enable reliable predictions of the aerodynamic and aeroacoustic performance of Contract Research Organization Reagent (CROR) propulsion systems. Tyler [19] demonstrated that the pressure field generated in the compressor consists of spinning mode patterns, and that the number of lobes in each pattern, together with the frequency of the pressure fluctuations, gives the speed at which the pattern spins. Additionally, there is a critical pattern speed which separates the subsequent behavior of the pattern into one of two different categories.

Yonezawa [20] studied the phase resonance phenomenon in centrifugal rotating machinery, compared and analyzed the phase resonance of the working condition of the pump and turbine, respectively, and revealed the important influence of the direction of rotor-stator interaction and radial velocity fluctuation on the phase resonance. Shigemitsu [21] studied the performance and internal flow of a small centrifugal pump with a large blade outlet angle through experiments and numerical simulation by using a shunt blade. Through experiments and numerical analysis, Cavazzini [22] compared the cavitation performance of centrifugal pumps with and without diverging blades and found that the cavitation allowance of the impeller with splitter blades decreased significantly at high flow rates, while the cavitation allowance increased significantly at low flow rates. Iwadachi [23] introduced a kind of turbine runner with a long and short blade applied to the adjustable speed setting. By improving the operation efficiency of partial loads, the stable operation range under the speed regulation mode was expanded.

In this paper, the frequency of interference is predicted theoretically, and several monitoring points are set up in different components and locations of the pump-turbine. The pressure fluctuation characteristics of the pump-turbine with splitter blades under different flow rates are analyzed based on the CFD numerical calculation method and combined with relevant test data, which provides certain theoretical reference for the design and improvement of pump-turbines.

\section{Numerical Simulation and Tests}

\subsection{Description of Geometry Models}

The model of the pump-turbine with splitter blades in this study was of a 1:7.47 scale for a pump-turbine in a domestic pumped storage power station. Basic parameters of the pump-turbine model are shown in Table 1.

Table 1. Basic parameters of the pump-turbine model.

\begin{tabular}{cc}
\hline Parameter & Data \\
\hline Number of stay vanes & 16 \\
Number of guide vanes & 16 \\
Number of impeller vane & 10 \\
Impeller outlet minimum diameter D1 $(\mathrm{mm})$ & 300 \\
Impeller outlet maximum diameter D2 $(\mathrm{mm})$ & 584 \\
runner outlet diameter $(\mathrm{mm})$ & 55 \\
volute outlet diameter $(\mathrm{mm})$ & 320 \\
Draft tube outlet diameter $(\mathrm{mm})$ & 600 \\
\hline
\end{tabular}

This paper studies the characteristics of the pump-turbine in the working condition of the pump. The working condition of the pump is as follows: First, the runner rotates anticlockwise, and water enters the draft tube. After passing through the runner, it is guided into the volute by the double-row cascade with a stay guide vane. Then, the casing converts the kinetic energy of the fluid to static pressure 
energy, so as to pump the water to the upstream reservoir. In this paper, Creo software is used to carry out 3D modeling for each part. The main flow components of a pump-turbine include the volute, stay vanes, guide vanes, runner, and draft tube. The model of the full flow geometric is shown in Figure 1 and the meshes of different components are shown in Figure 2.

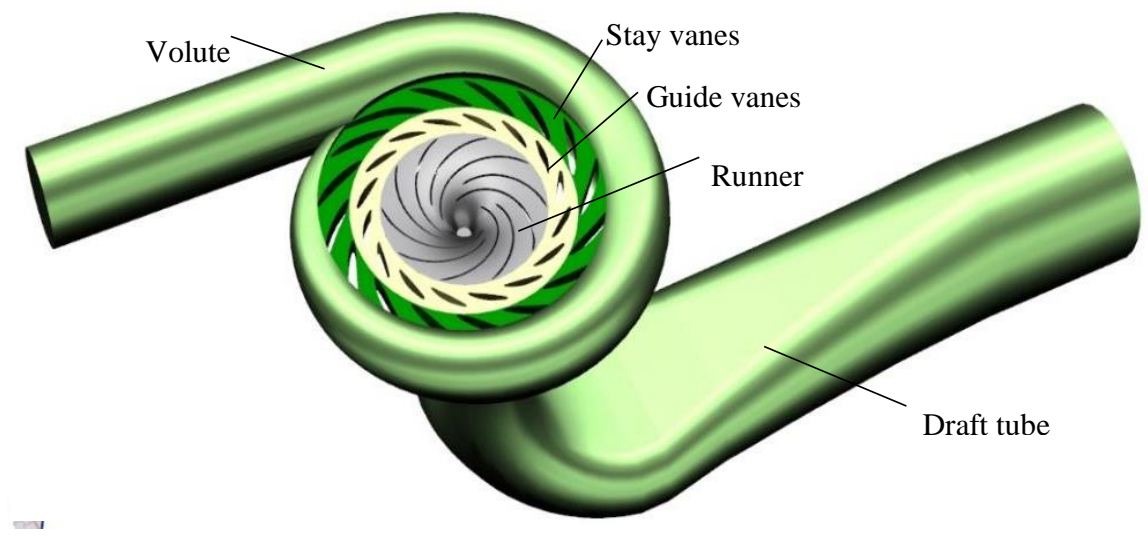

Figure 1. Full flow geometric.

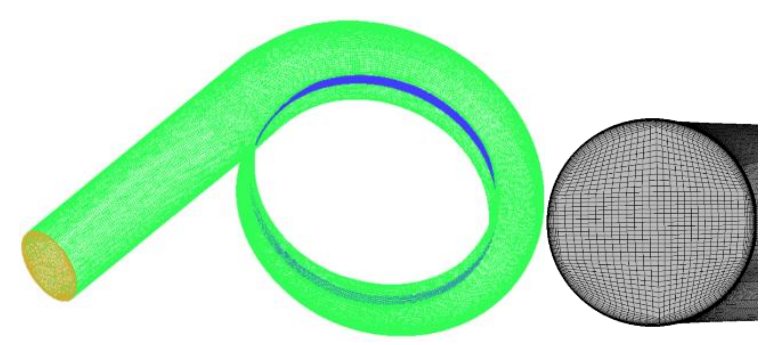

(a) Mesh of volute passage.

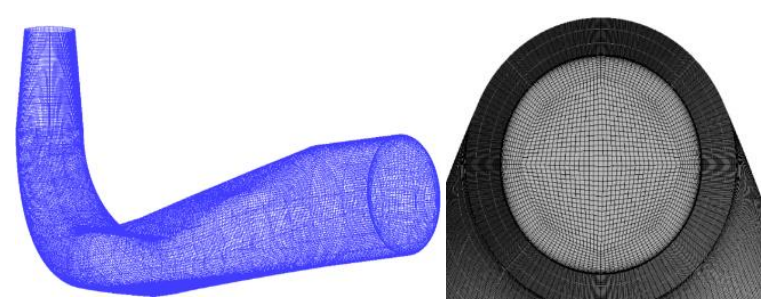

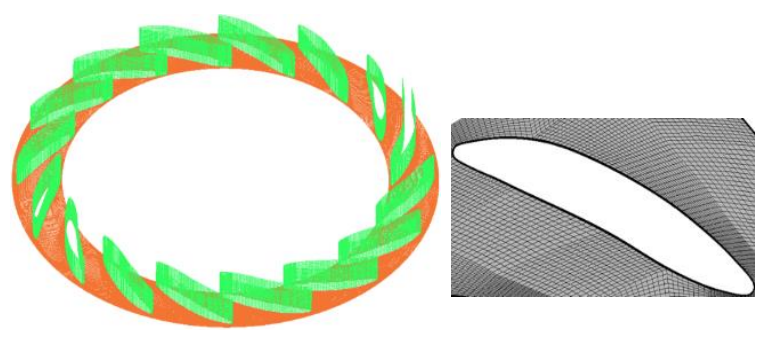

(b) Mesh of stay vane passage.

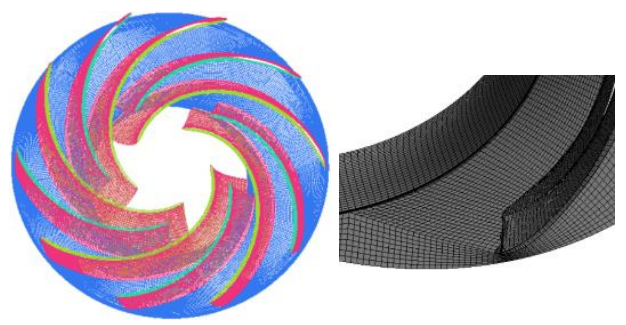

(c) Mesh of draft tube passage. (d) Mesh of runner channel.

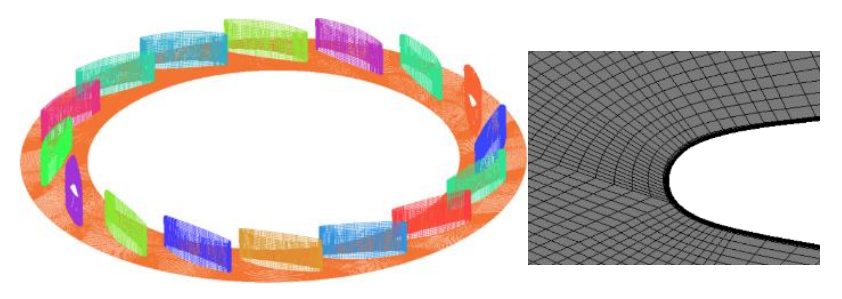

(e) Mesh of guide vane passage

Figure 2. Mesh.

\subsection{Grid and Independence Analysis}

The characteristic of the structured grid is that the nodes can be named in order with fixed rules, which means that the computation is small, the grid quality is easy to control, the boundary layer grid is guaranteed, and the convergence of computation can be improved. In this paper, considering the 
geometric characteristics of the pump-turbine model and calculation accuracy requirements, ICEM CFD software was used to divide the full flow passage into five parts (the volute, stay vanes, guide vanes, draft tube and runner), then converted into hexahedral meshes. The initial block was cut and O-type subdivided to form a decagonal shape, which is a better mapping of the curved section. Then, the node was combined with the array block. The quality of the generated structured grids was higher than 0.5, and the angle was greater than $25^{\circ}$. Meshes of each part are shown in Figure 3.

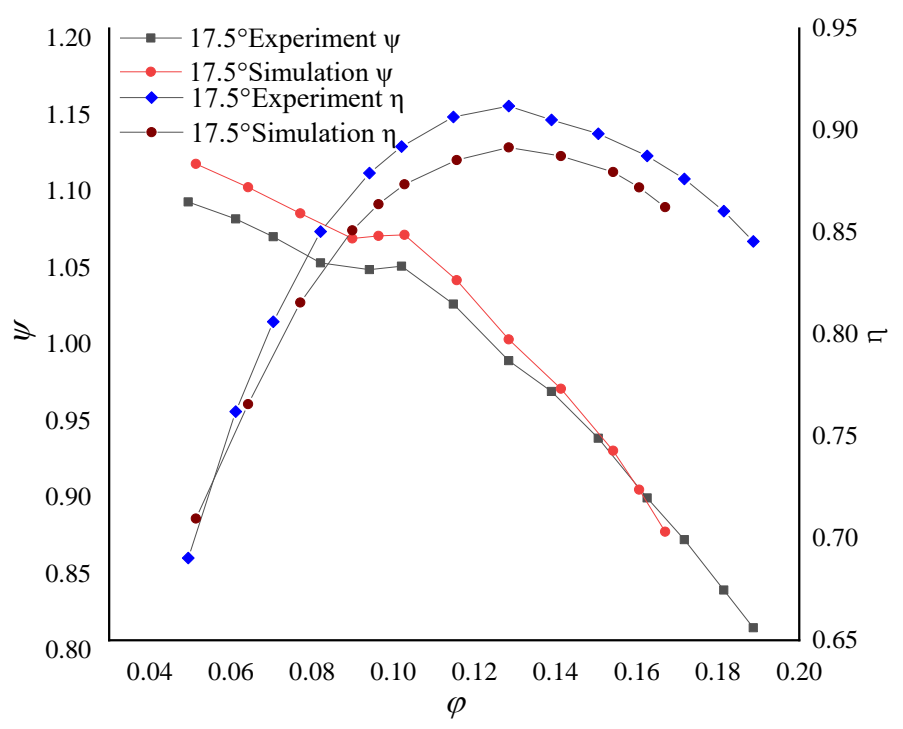

Figure 3. Comparison of performance between simulation and experiment.

Since the number of grids has a great impact on the calculation results, the grid number independence analysis was performed for five sets of grids. According to the rated operating condition, with guide vanes set to openings of $17.5^{\circ}$, eight groups of grid number schemes were set at uniform intervals between 4 million and 10 million.

It can be seen from Table 2 that with the increase of the total number of grids, the simulation results tended to be stable. When the total number of grids reached 7.2 million or more, the simulated relative error rate on the head changes very little, so it can be considered that the number of grids has little influence on the calculation results. Considering the computing resources and solution accuracy, Case4 was selected, and the total number of grids was 7,213,000.

Table 2. Influence of number of grid cells on accuracy.

\begin{tabular}{|c|c|c|c|c|c|c|c|}
\hline & $\begin{array}{c}\text { Volute } \\
\left(10^{6}\right)\end{array}$ & $\begin{array}{c}\text { Stay } \\
\text { Vanes } \\
\left(10^{6}\right)\end{array}$ & $\begin{array}{c}\text { Guide } \\
\text { Vanes } \\
\left(10^{6}\right)\end{array}$ & $\begin{array}{l}\text { Draft } \\
\text { Tube } \\
\left(10^{6}\right)\end{array}$ & $\begin{array}{c}\text { Runner } \\
\left(10^{6}\right)\end{array}$ & $\begin{array}{l}\text { Total } \\
\left(10^{6}\right)\end{array}$ & $\begin{array}{l}\Delta \mathrm{H} \\
(\%)\end{array}$ \\
\hline Case1 & 0.486 & 0.883 & 0.982 & 0.606 & 1.309 & 4.266 & 2.732 \\
\hline Case2 & 0.651 & 1.102 & 1.197 & 0.775 & 1.459 & 5.184 & 2.093 \\
\hline Case3 & 0.845 & 1.297 & 1.364 & 1.009 & 1.638 & 6.153 & 1.285 \\
\hline Case4 & 1.076 & 1.528 & 1.589 & 1.176 & 1.844 & 7.213 & 0.828 \\
\hline Case5 & 1.260 & 1.732 & 1.771 & 1.353 & 2.065 & 8.181 & 0.826 \\
\hline Case6 & 1.492 & 1.916 & 1.965 & 1.548 & 2.281 & 9.202 & 0.825 \\
\hline Case7 & 1.707 & 2.097 & 2.166 & 1.733 & 2.467 & 10.170 & 0.824 \\
\hline
\end{tabular}

The numerical calculations for different schemes were performed with the fluid simulation code ANSYS CFX, using the unsteady Reynolds-averaged Navier-Stokes equation, which is based on the finite volume method 14. The turbulence inside the pump was simulated by means of the shear stress transport (SST) model, and the reference pressure was set to 101,325 Pa. The constant total pressure and mass flow rate were given at the pump inlet and outlet as boundary conditions, respectively, since 
the water enters radially and leaves axially. The frozen rotor setting was imposed on the interface dynamic and static components to simulate real relative position movement. Besides that, mass and momentum were set to no slip, and the wall roughness was set to the smooth wall setting. The advection scheme was set to high resolution and upwind. The solution convergence criterion was set to $10^{-5}$. The Scale-adaptive simulation (SST - SAS) model was adopted for unsteady calculation, and the initial value of the unsteady calculation was the steady calculation result of the corresponding working conditions. The MRF model of multi-reference coordinate systems was adopted for the simulation of dynamic and static components. Rotor-stator interaction was imposed on the interface between the runner, the volute, and the draft tube. The impeller rotation time of $2^{\circ}$ was taken as a time step, and the calculated time selected was 10 rotation cycles.

\subsection{Experiment Validations on External Characteristics}

Experimental measurement and CFD numerical calculation methods were used to study and analyze the external characteristics of the pump-turbine with splitter blades. The flow rate and head are normalized as flow rate coefficient $\varphi$ and the head coefficient by the following equations:

$$
\begin{gathered}
\psi=\frac{2 g H}{u_{2}^{2}} \\
\varphi=\frac{Q}{u_{2} R_{2}^{2}}
\end{gathered}
$$

where $H$ is the head, $u_{2}$ is the outlet circumferential velocity of the runner, $g$ is the acceleration of gravity, $Q$ is the flow rate, and $R_{2}$ is the radius of the high pressure side of the runner.

The pump-turbine model tests were carried out at the Hydraulic Machinery Laboratory of École Polytechnique Fédérale de Lausanne (EPFL). The EPFL Hydraulic Machinery Laboratory is equipped with three closed circulation type universal hydraulic machinery test stands. In accordance with the requirements of IEC60193, a high-precision test instrument was installed on the test bench. The test bench can carry out research and development and test vertical or horizontal hydraulic machinery such as pumps, turbines, and pump-turbines. On the basis of this experimental bench, the head and efficiency of the pump-turbine were measured and recorded.

Figure 3 shows the comparison of performance between the simulation and the experiment. Both the simulation head and efficiency show good agreement with the experiment. The maximum relative error rate on head in each condition was less than $4.5 \%$, and the maximum relative error on efficiency was less than $3.2 \%$, indicating that the simulation calculation can accurately predict the performance of the pump-turbine model in working pump conditions and ensure the accuracy of further analysis.

\section{Setting and Theoretical Prediction}

\subsection{Setting of Monitoring Points}

In order to obtain the unsteady fluctuation distribution and propagation characteristics in the full flow passage, a number of monitoring points were selected at different locations of each component. Figure 4 shows 12 monitoring points on the intermediate surface of the draft tube. The points at the inlet of the draft tube are denoted as DT01-03, the points at the inlet of the straight cone tube are denoted as DT04-06, the points at the elbow tube are denoted as DT07-09, and the points at the inlet of the draft tube are denoted as DT10-12. Figure 5 shows the 20 monitoring points on the middle flow surface of the runner. The points from the outlet to the inlet near the pressure surface of the long blade are denoted as RN01-05, the points from the outlet to the inlet near the suction surface of the short blade are denoted as RN06-10, the points from the outlet to the inlet near the short blade pressure surface are denoted as RN11-15, and the points from the outlet to the inlet near the suction surface of the long blade are denoted as RN16-20. Figure 6 shows 160 monitoring points in different sections 
(span0.1 for the top, span0.5 for the middle, and span0.9 for the bottom) of the double-row cascade flow passage, and $48 \times 3$ points were distributed in the circumferential direction of the bladeless region, which are denoted as GR001-GR048 (span0.5), GR101-GR148 (span0.1), and GR001-GR048 (span0.9) in a counterclockwise direction. Four monitoring points were arranged in each span 0.5 section of four different guide vane passages, totaling 16 points. According to the serial number of the passage, they are GV001-GV004 (guide vane passage), SG001-SG004 (mid-span between the guide vanes and stay vanes), SV001-SV004 (stay vane passage), and SS001-SS004 (stay vane exit). Figure 7 shows 12 monitoring points arranged on the middle surface of the volute flow passage according to the flow passage profile, which are denoted as SC01-SC12 from the tongue to the outlet.

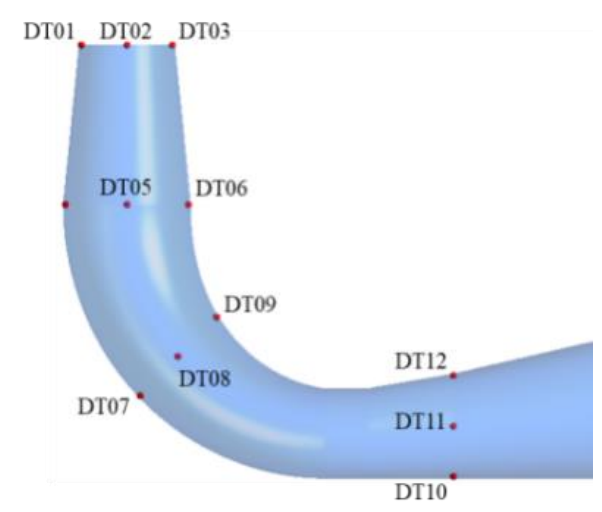

Figure 4. Monitoring points in the draft tube.

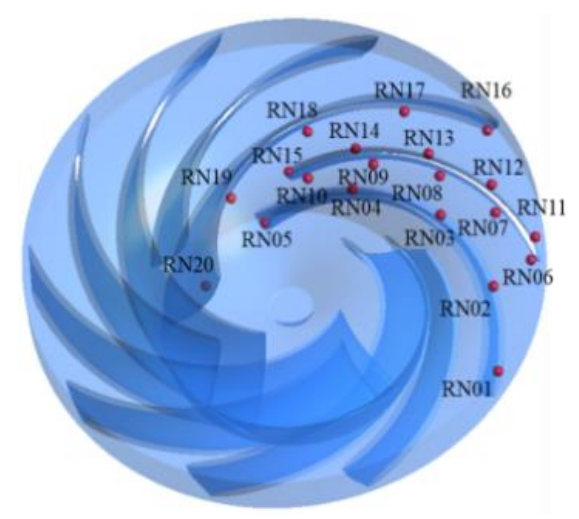

Figure 5. Monitoring points in the runner.
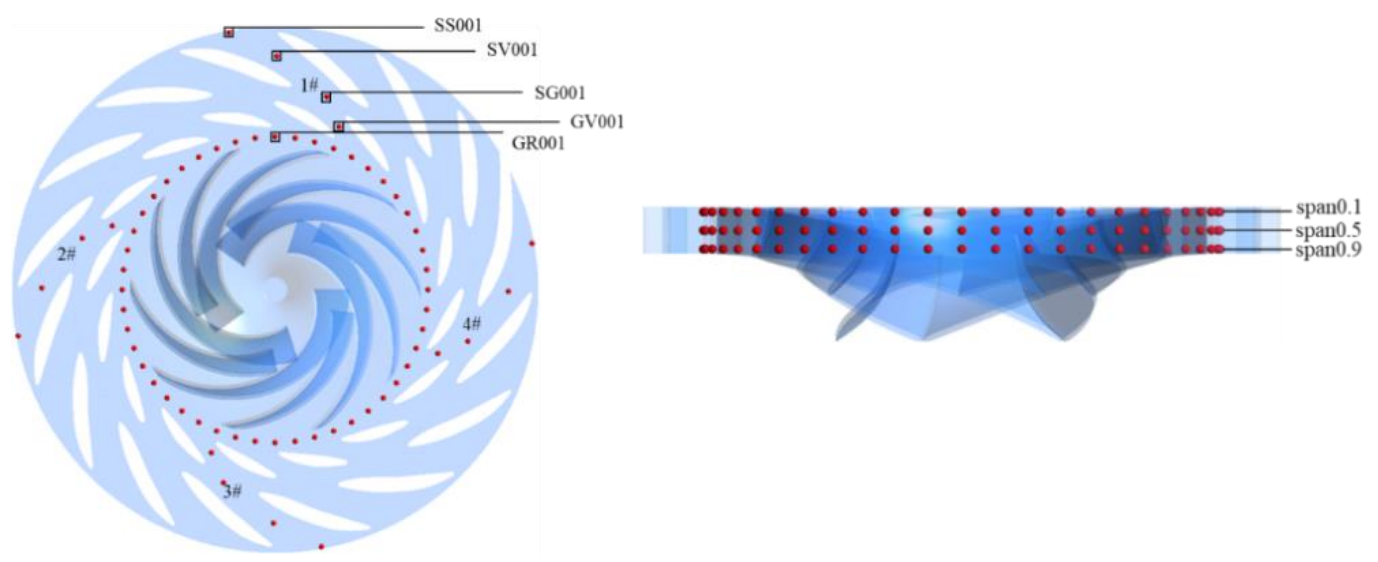

Figure 6. Monitoring points in the stay guide vanes. 


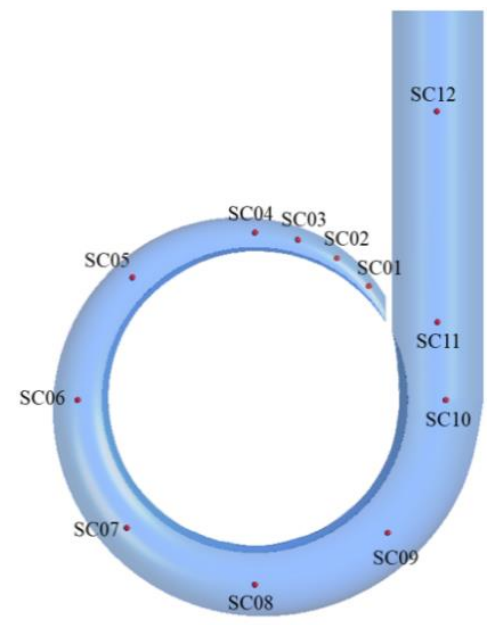

Figure 7. Monitoring points in the volute.

\subsection{Theoretical Prediction}

The rotor-stator interaction between the runner and the guide vanes is the main reason for the high frequency fluctuation in the runner. In this paper, according to the diameter modulus theory proposed by Kubota [24] and the method for hydraulic excitation in the pump-turbine, analyzed by Tanaka [25], considering the special structure of the long and short blades, the relatively prominent rotor-stator interaction frequency in the pump-turbine with long and short blades is predicted from the theoretical level.

Low-order and high-order hydraulic excitation modes can be induced by the rotor-stator interaction effect, dominated by potential flow. The modes can be expressed by the node diameter number $k$. The expressions of the minimum and maximum node diameters are as follows:

$$
\begin{aligned}
& k_{1}=m \cdot Z_{r}-n \cdot Z_{g} \\
& k_{2}=m \cdot Z_{r}+n \cdot Z_{g}
\end{aligned}
$$

where $m$ and $n$ are arbitrary integers, $Z_{r}$ is the number of runner blades $(5+5)$, and $Z_{g}$ is the number of guide vanes (16).

The number of high-pressure and low-pressure regions in the circumferential direction of a specific frequency is represented by $k$, whose pressure distribution is affected by the rotation of the runner. It also has a specific rotation speed. The angular velocity is calculated as follows.

In a stationary system, the calculation is

$$
\begin{aligned}
& \omega_{1}=m \cdot Z_{r} \cdot \omega / k_{1} \\
& \omega_{2}=m \cdot Z_{r} \cdot \omega / k_{2}
\end{aligned}
$$

In a rotating system, the calculation is

$$
\begin{aligned}
& \omega_{1}=n \cdot Z_{g} \cdot \omega / k_{1} \\
& \omega_{2}=n \cdot Z_{g} \cdot \omega / k_{2}
\end{aligned}
$$

where $\omega$ is the speed of the runner.

It can be seen from Equations (5-8) that the positivity of $k$ determines the rotation direction of the pressure field. When $k$ is positive, the rotation direction is consistent with the rotation direction of the runner, and vice versa. The action frequency of hydraulic excitation, caused by rotor-stator interaction on the runner, is shown as the guide vane overcurrent frequency $f_{r}$ and its frequency multiplication, 
while the action frequency in the guide vane stationary domain is shown as the overcurrent frequency $f_{s}$ and its frequency multiplication of the runner blade. The expression is as follows:

$$
\begin{aligned}
& f_{r}=n \cdot Z_{g} \cdot f_{n} \\
& f_{s}=m \cdot Z_{r} \cdot f_{n}
\end{aligned}
$$

Table 3 shows the rotor-stator interaction frequencies at different node-diameter numbers. In general, the greater the absolute value of $k$, the smaller the corresponding amplitude, and the smaller the influence of rotor-stator interaction. The frequency corresponding to the smaller absolute value $\mathrm{k}_{1}$ is listed in the table, and the minimum diameter $\mathrm{k}_{1}$ was $2,-2,4$, and -6 , in order from the smallest absolute value to the largest. Although the absolute value was smallest when $\mathrm{k}_{1}= \pm 2$, the corresponding frequency was larger, and the amplitude was lower than that when $\mathrm{k}_{1}=4$ and -6 . Therefore, the rotor-stator interaction frequency acting in the static system was mainly $10 f_{n}$ and $20 f_{n}$, and the rotation speeds of their respective pressure fields were $-1.67 \omega$ and $5 \omega$, respectively. Its amplitude cannot be determined in theory, so we need to judge it according to the calculation results. The rotor-stator interaction frequency in the rotation system was mainly $16 \mathrm{f}_{\mathrm{n}}$, but the pressure field under a different node-diameter number had two different kinds of rotation speed, $-2.67 \mathrm{\omega}$ and $4 \omega$. In addition, due to the special design of long and short blades, flows at the outlet of long blades and short blades are significantly different, leading to differences in rotor-stator interaction between stay guide vanes. Thus, $5 \mathrm{f}_{\mathrm{n}}$ and its frequency doubling of a single type of blade can be generated.

To sum up, in theory, the more prominent rotor-stator interaction frequencies in the stationary system are $5 f_{n}, 10 f_{n}$, and $20 f_{n}$, and the more prominent rotor-stator interaction frequencies in the rotating system are $16 \mathrm{f}_{\mathrm{n}}$. However, the accuracy of the prediction results and the specific situation need to be judged from the calculation results.

Table 3. Frequency of rotor-stator interaction under different pitch moduli.

\begin{tabular}{ccccccc}
\hline \multirow{2}{*}{$\mathbf{n}$} & $\mathbf{m}$ & $\mathbf{k}_{\mathbf{1}}$ & \multicolumn{2}{c}{ Stationary System } & \multicolumn{2}{c}{ Rotating System } \\
\cline { 3 - 7 } & & & $\boldsymbol{\omega}_{\mathbf{1}} / \boldsymbol{\omega}$ & $\mathbf{f}_{\mathbf{s}} / \mathbf{f}_{\mathbf{n}}$ & $\boldsymbol{\omega}_{\mathbf{1}} / \boldsymbol{\omega}$ & $\mathbf{f}_{\mathbf{r}} / \mathbf{f}_{\mathbf{n}}$ \\
\hline 1 & 1 & -6 & -1.67 & 10 & -2.67 & 16 \\
1 & 2 & 4 & 5 & 20 & 4 & 16 \\
2 & 3 & -2 & -15 & 30 & -16 & 32 \\
3 & 5 & 2 & 25 & 50 & 24 & 48 \\
\hline
\end{tabular}

\subsection{Experiment Validations on Pressure Fluctuation}

In order to prove the reliability of the numerical calculation of pressure fluctuation, this paper compared and analyzed the calculation and test results of the monitoring points near the bottom ring in the bladeless region under the rated conditions. The degree of pressure fluctuation is expressed by the dimensionless number $d H / H$ (where $d H$ is the peak of pressure fluctuation and $H$ is the head). Table 4 shows the protruding frequency and corresponding amplitude of pressure fluctuation in the bladeless region after fast Fourier transform (FFT) transformation and the comparison with the experimental results. It can be seen from the table that the protruding frequencies of the pressure fluctuation in the bladeless region were $10 \mathrm{f}_{n}, 20 \mathrm{f}_{n}$, and $5 \mathrm{f}_{n}$, in descending order according to the amplitude, which was the same as the theoretical prediction of the rotor-stator interaction frequency above, indicating that the pressure fluctuation in the bladeless region is mainly caused by rotor-stator interaction. It can be seen from the relative error of the corresponding amplitude that the unsteady numerical calculation overpredicted the amplitude of $10 f_{n}$ and $20 f_{n}$ at the node-diameter number $\mathrm{k}_{1}=-6$ and 4 , but underpredicted the amplitude of $5 \mathrm{f}_{\mathrm{n}}$. This is because the compressibility of the fluid was ignored in the numerical calculation, the flow difference near the long and short blades was not sufficiently captured, and the influence of the flow solid coupling was not considered in the actual 
situation. In general, unsteady pressure fluctuation calculation can accurately capture the rotor-stator interaction frequency, and the error of the corresponding amplitude is also within an acceptable range. Therefore, the reliability of the numerical calculation of pressure fluctuation in this paper can be proven.

Table 4. Comparison between calculation and test results of pressure fluctuation in the bladeless region.

\begin{tabular}{cccc}
\hline Frequency & $\begin{array}{c}\text { Experimental Value } \\
d H / H(\%)\end{array}$ & $\begin{array}{c}\text { Calculated Value } \\
d H / H(\%)\end{array}$ & Relative Error (\%) \\
\hline $5 \mathrm{f}_{\mathrm{n}}$ & 0.989 & 0.887 & -10.31 \\
$10 \mathrm{f}_{\mathrm{n}}$ & 2.068 & 2.322 & +12.28 \\
$20 \mathrm{f}_{\mathrm{n}}$ & 1.425 & 1.594 & +11.86 \\
\hline
\end{tabular}

\section{Results and Discussions}

\subsection{Pressure Fluctuation Analysis of Runner}

Figure 8 shows pressure fluctuation distributions at the mid-span of the runner under different flow rates within five rotation cycles. It can be seen from the figure that the pressure fluctuation of the runner from the inlet to the outlet gradually increased. The fluctuation near the trailing edge pressure surface of the splitter blades was the most intense, due to the influence of rotor-stator interaction. The pressure fluctuation intensity at a small flow rate at $0.75 \mathrm{Q}_{\mathrm{d}}$ was large, and the pressure fluctuation on both sides of the trailing edge of the splitter blades was particularly significant. With the increase of flow, the pressure fluctuation inside the runner decreased gradually, and the strong fluctuation region was mainly concentrated in the pressure surface at the trailing edge of the splitter blades. This indicates that, at the same opening and under the condition of a small flow, the flow instability inside the runner leads to a greater intensity of internal pressure fluctuation and intensifies the rotor-stator interaction at the trailing edge of the blades. Moreover, the fluctuation near the pressure surface at the trailing edge of the splitter blades and the suction surface is particularly violent under a small flow. Additionally, the flow pattern inside the runner will be improved when the flow rate increases, and the rotor-stator interaction at mid-span in the runner and the stay guide vanes will also be improved to some extent, thus reducing the pressure fluctuation intensity inside the runner, especially at the suction surface of the trailing edge of the blades.

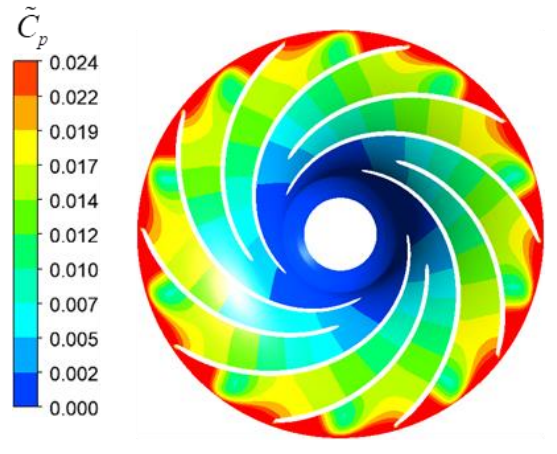

(a) $0.75 Q_{d}$

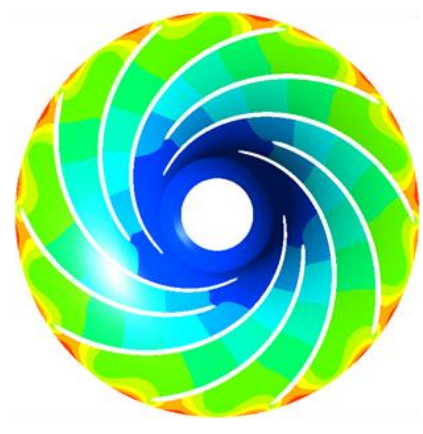

(b) $1.0 \mathrm{Qd}_{\mathrm{d}}$

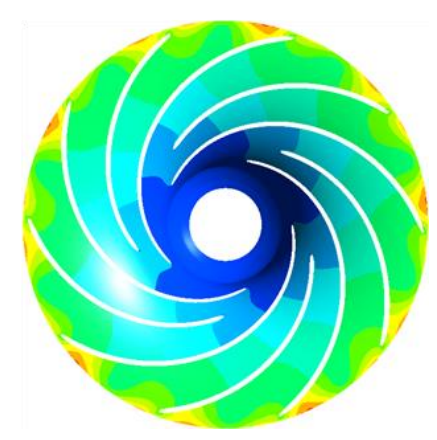

(c) $1.25 \mathrm{Q}_{\mathrm{d}}$

Figure 8. Pressure fluctuation distributions at the mid-span of the runner.

Figures 8-11 show the frequency domain of pressure fluctuation near both sides of the long and short blades through FFT transform processing. The $x$ coordinate is the frequency ( $f$ is the actual frequency in the figure and $\mathrm{f}_{\mathrm{n}}$ is the axial frequency), and the $y$ coordinate is the names of the monitoring points near the blades. According to the pressure fluctuation coefficient $C_{p}$, the color reflects the frequency domain of pressure fluctuation and its amplitude variation trend. 
Figures 9 and 10 show the frequency domain of pressure fluctuation near the pressure surface and suction surface of the long blade under different flow rates at monitoring points RN01 RN05 and RN16 RN20, respectively. Figure 8 shows that the frequency domain of pressure fluctuation near the pressure side of the long blade decreased gradually from the blade outlet to the inlet and changed with different flow rates. Under a small flow rate at $0.75 \mathrm{Q}_{d}$, there was also a nonlinear frequency with a high amplitude between $9 f_{n}$ and $10 f_{n}$, whose main frequency was about $9.2 f_{n}$, in addition to the guide vane passage frequency of $16 \mathrm{f}_{\mathrm{n}}$ caused by rotor-stator interaction. The main reason for this phenomenon is that there was a strong flow instability under the working conditions. Under the rated flow rate at $1.0 Q_{d}$, the amplitude of pressure fluctuation decreased, especially for the fluctuation corresponding to the nonlinear frequency. The main frequency was the guide vane passage frequency of $16 \mathrm{f}_{\mathrm{n}}$, and the low-axis frequency doubling was $\mathrm{f}_{\mathrm{n}} \sim 4 \mathrm{f}_{\mathrm{n}}$. As the monitoring points became far away from the guide vane, the amplitude of $16 \mathrm{f}_{\mathrm{n}}$ attenuated rapidly, and its main frequency gradually changed from $16 f_{n}$ to $f_{n}$ from the blade outlet to the inlet. By comparing Figure 9 with Figure 10, it can be seen that the frequency domain distribution of pressure fluctuation near both sides of the long blade was similar, but the amplitudes were different. Compared with RN01, the amplitude of the guide vane passage frequency $16 \mathrm{f}_{\mathrm{n}}$ at RN16 decreased by about $42.6 \%, 44.2 \%$, and $51.9 \%$, respectively from $0.75 \mathrm{Q}_{\mathrm{d}}$ to $1.25 \mathrm{Q}_{d}$, while the amplitude of $\mathrm{f}_{\mathrm{n}}$ only decreased by $-1.5 \%, 5.3 \%$, and $9.7 \%$, respectively. Therefore, the low-axis frequency doubling near the suction side of the long blade was more prominent. Above, the flow condition inside the runner can be improved by increasing the flow rate. The amplitude of nonlinear frequency was greatly reduced, and the main frequency was the axial frequency doubling caused by rotor-stator interaction.

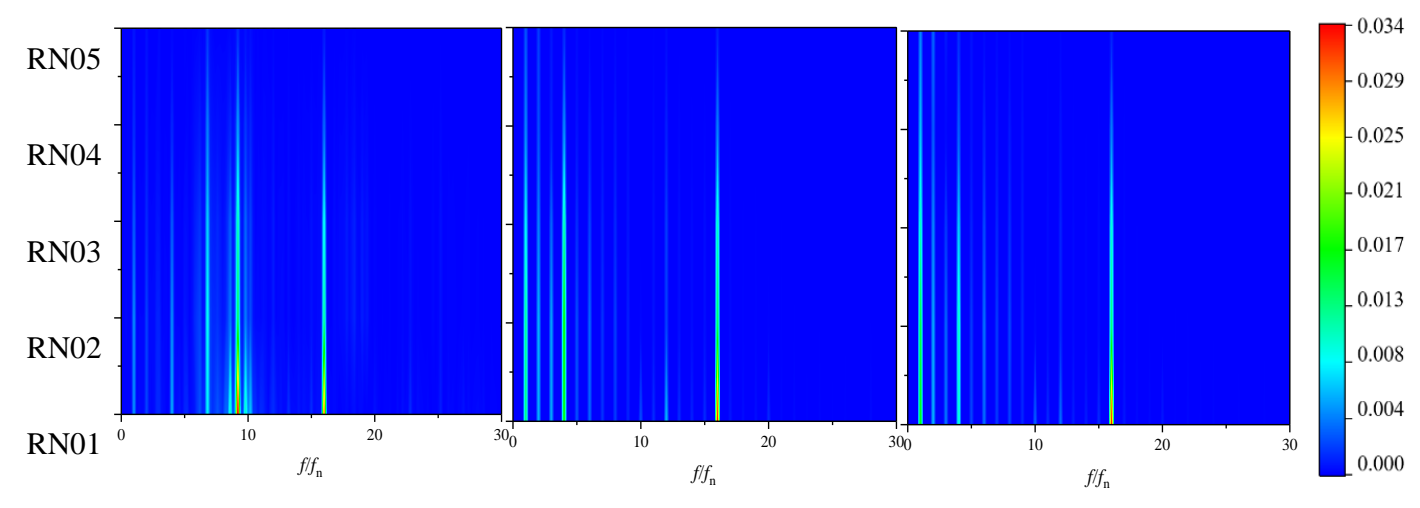
(a) $0.75 Q_{d}$
(b) $1.0 \mathrm{Q}_{\mathrm{d}}$
(c) $1.25 Q_{d}$

Figure 9. Frequency domain of pressure fluctuation near the pressure side of the long blade.

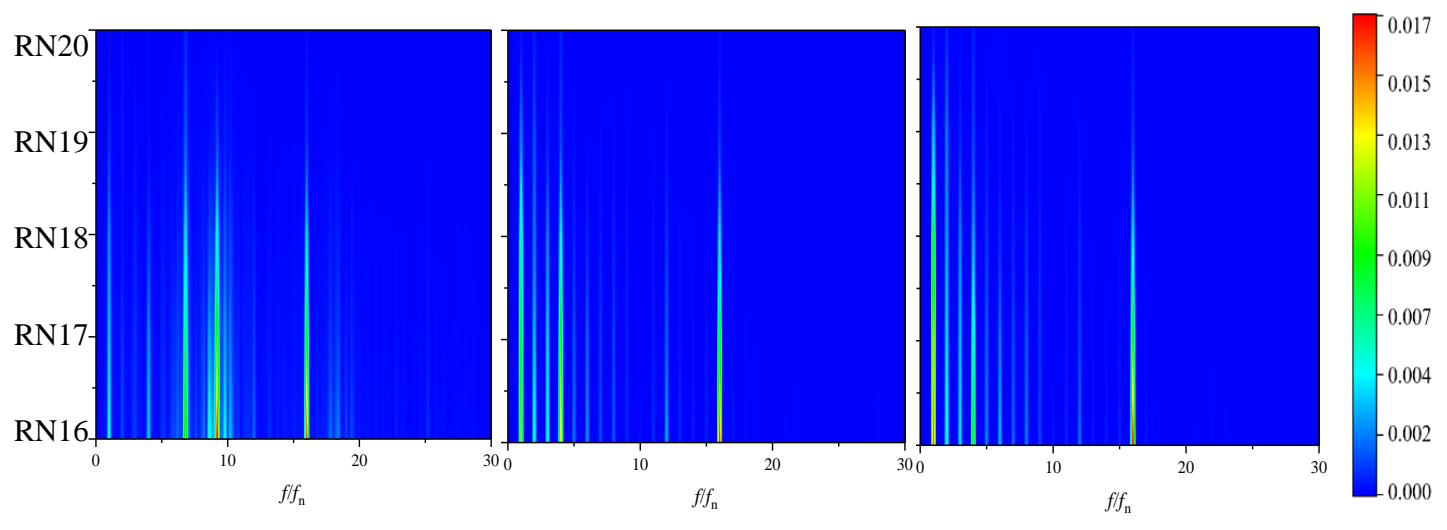
(a) $0.75 Q_{d}$
(b) $1.0 \mathrm{Q}_{\mathrm{d}}$
(c) $1.25 \mathrm{Qd}_{\mathrm{d}}$

Figure 10. Frequency domain of pressure fluctuation near the pressure side of the short blade. 
Figures 11 and 12 show the frequency domain of pressure fluctuation near the pressure surface and suction surface of the short blade under different flow rates at the monitoring points RN11 RN15 and RN06 RN10, respectively. Compared with the figures above, it can be seen that the frequency domain distribution of pressure fluctuation of the long and short blades was similar, but the fluctuation amplitude of the pressure side was reduced, while that of the suction side increased at the monitoring points of the short blade. In addition, under the influence of potential flow interference at the outlet of the adjacent long blades, the amplitude of the guide vanes passing through frequency $16 \mathrm{f}_{\mathrm{n}}$ near the pressure side of the short blade attenuated from the outlet of the blades, but there was an obvious surge at RN13, which was at the middle position of the pressure surface. Compared with RN12, the amplitude of $16 f_{n}$ at RN13 increased by about $89.8 \%, 63.6 \%$, and $61.5 \%$, respectively from 0.75 $\mathrm{Q}_{\mathrm{d}}$ to $1.25 \mathrm{Q}_{\mathrm{d}}$, which indicates that the influence of the outlet potential flow of the long blade on the pressure surface of the short blade was more significant under a small flow rate.

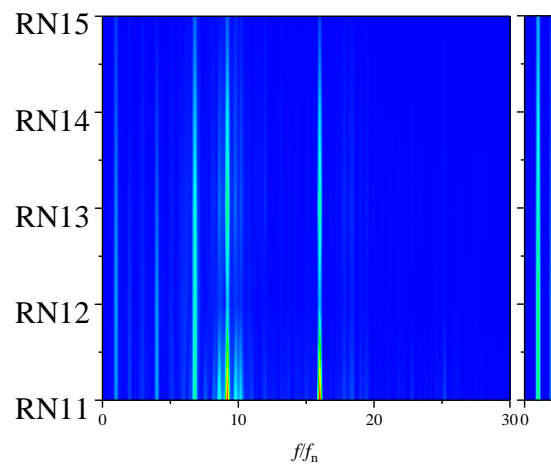

(a) $0.75 Q_{d}$

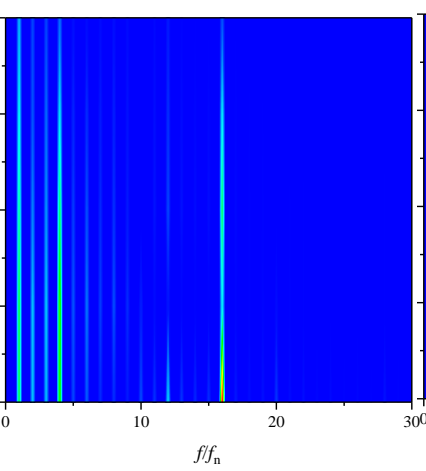

(b) $1.0 \mathrm{Q}_{\mathrm{d}}$

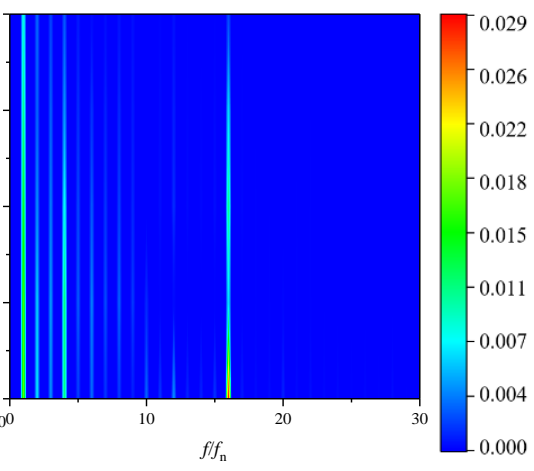

(c) $1.25 \mathrm{Qd}_{\mathrm{d}}$

Figure 11. Frequency domain of pressure fluctuation near the pressure side of the short blade.

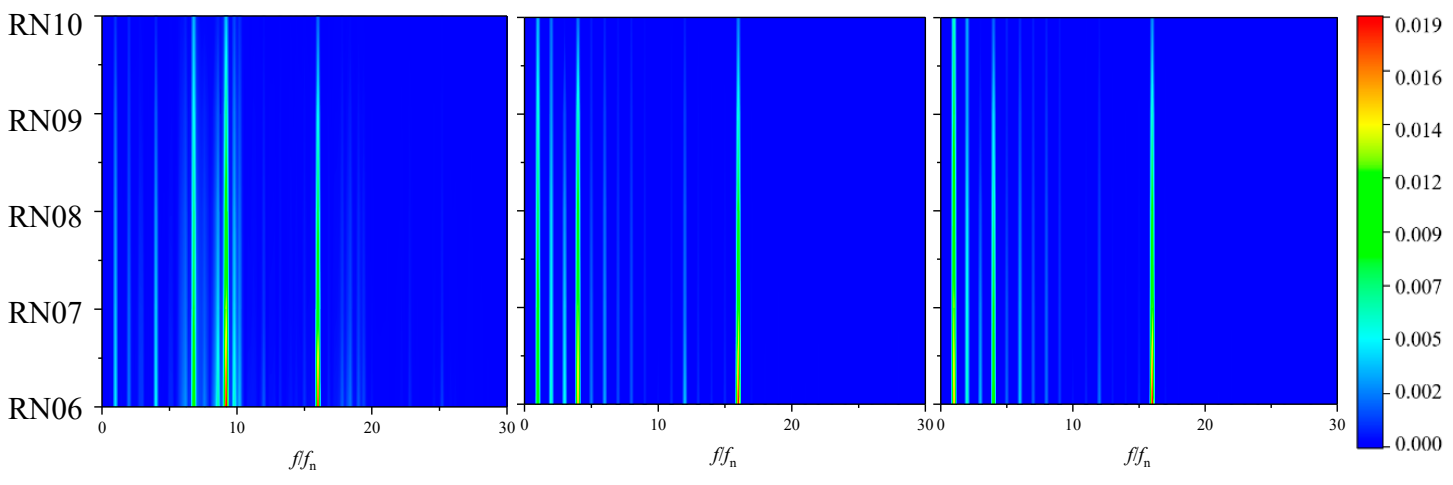
(a) $0.75 \mathrm{Q}_{\mathrm{d}}$
(b) $1.0 Q_{\mathrm{d}}$
(c) $1.25 \mathrm{Q}_{\mathrm{d}}$

Figure 12. Frequency domain of pressure fluctuation near the suction side of the short blade.

\subsection{Pressure Fluctuation Analysis of Bladeless Region}

The bladeless region between the runner and the stay guide vane is the area with the strongest rotor-stator interaction. In order to obtain the unsteady fluctuation characteristic of its flow field, 48 monitoring points were uniformly arranged in the circumferential direction. Figure 13 shows the time and frequency domain of pressure fluctuation of monitoring point GR001 under different flow rates. The time domain diagram on the left of the figure shows the variation of pressure fluctuation in a rotation period. The time domain distribution of pressure fluctuation under a rated flow rate and large flow rate had obvious periodicity, while under the small flow rate it was irregular due to flow instability. Figure 14 shows that there were many nonlinear frequencies under the small flow rate at 
$0.75 Q_{d}$, and the frequencies with high amplitudes included $5 f_{n}, 10 f_{n}$, and $20 f_{n}$, caused by rotor-stator interaction, as well as the main frequency of $7.8 \mathrm{f}_{\mathrm{n}}$. The frequencies under a rated flow rate at 1.0 $\mathrm{Q}_{\mathrm{d}}$ and large flow rate at $1.25 \mathrm{Q}_{\mathrm{d}}$ were mainly the axial frequency multiplier caused by rotor-stator interaction. The first, second, and third main frequencies at $1.0 Q_{d}$ were $10 f_{n}, 20 f_{n}$, and $5 f_{n}$, and the first, second, and third main frequencies at $1.25 Q_{d}$ were $20 f_{n}, 10 f_{n}$, and $5 f_{n}$, respectively. At the monitoring point, when the flow rate was at $1.0 \mathrm{Q}_{\mathrm{d}}$, the rotor-stator interaction was strong when the node-diameter number $\mathrm{k}_{1}=-6$. When the flow rate was at $1.25 \mathrm{Q}_{\mathrm{d}}$, the rotor-stator interaction was strong when the node-diameter number $k_{1}=4$. This indicates that the flow rate will affect the energy distribution of the rotor-stator interaction with different node-diameter numbers.

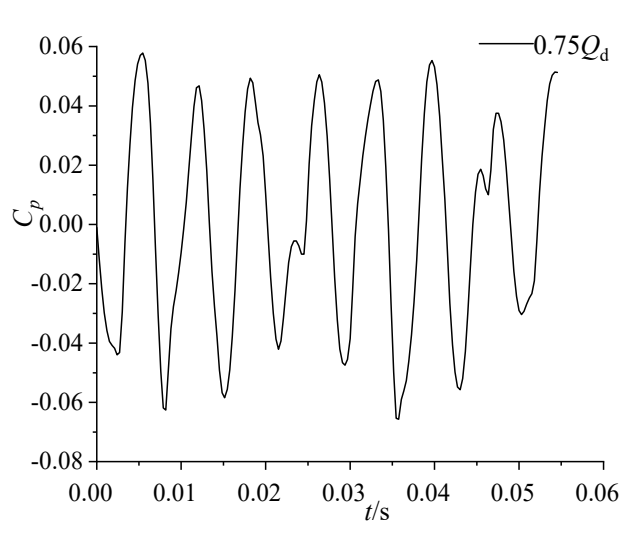

(a) Time domain at $0.75 \mathrm{Q}_{\mathrm{d}}$.

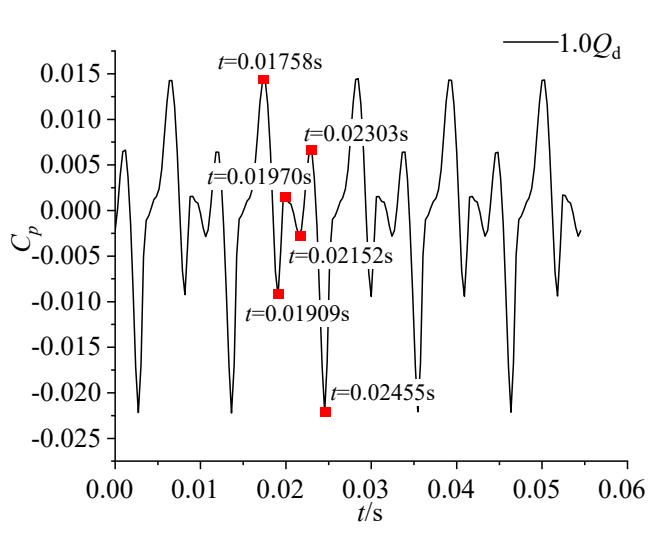

(c) Time domain at $1.0 \mathrm{Q}_{\mathrm{d}}$.

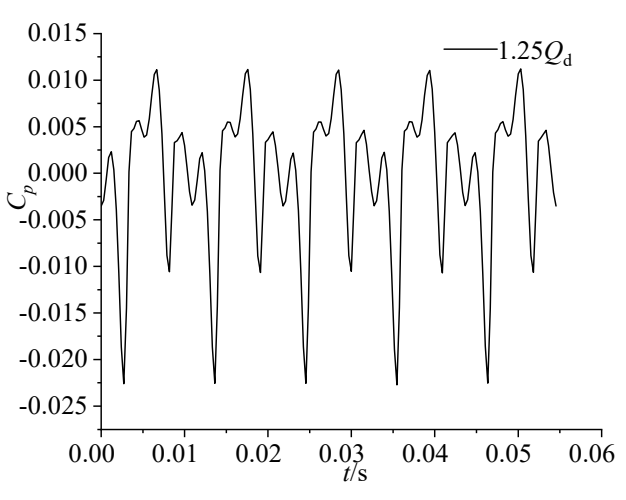

(e) Time domain at $1.25 \mathrm{Qd}$.

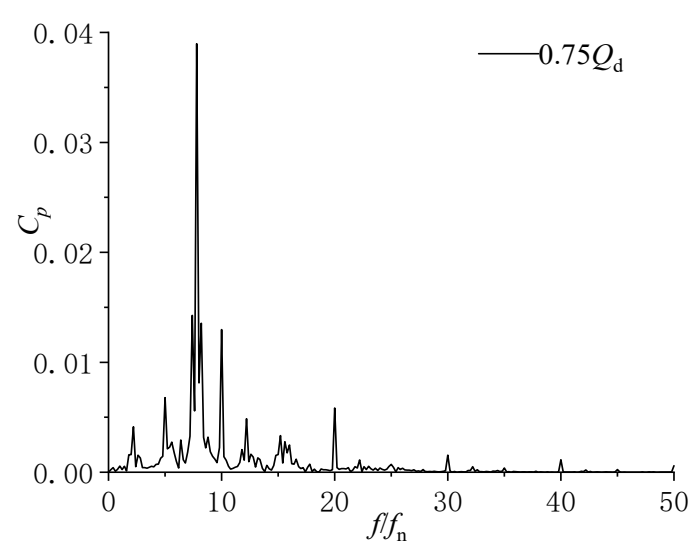

(b) Frequency domain at $0.75 \mathrm{Q}_{\mathrm{d}}$.

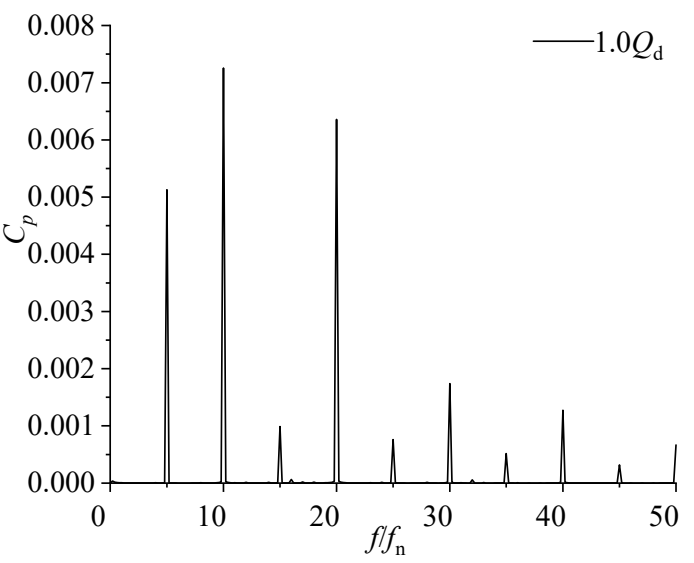

(d) Frequency domain at $1.0 \mathrm{Q}_{\mathrm{d}}$.

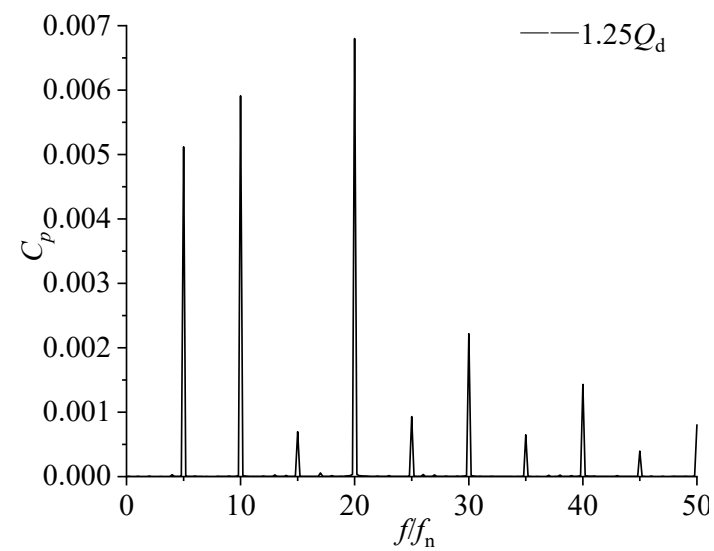

(f) Frequency domain at 1.25 Qd.

Figure 13. Time and frequency domain of pressure fluctuation of GR001. 

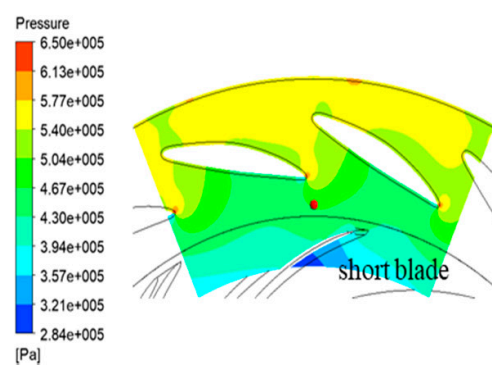

(a) $\mathrm{t}=0.01758 \mathrm{~s}$.
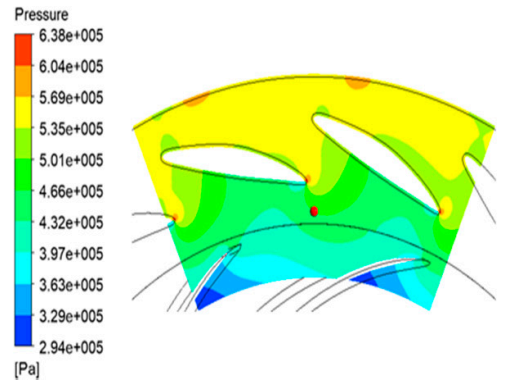

(d) $\mathrm{t}=0.02152 \mathrm{~s}$.
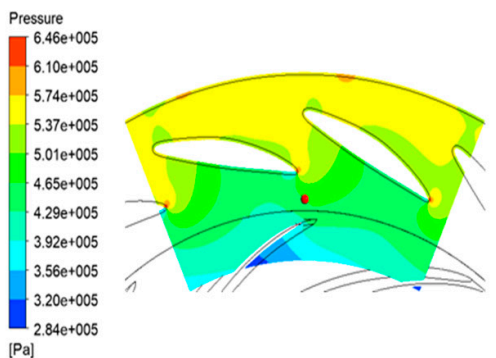

(b) $\mathrm{t}=0.01909 \mathrm{~s}$.
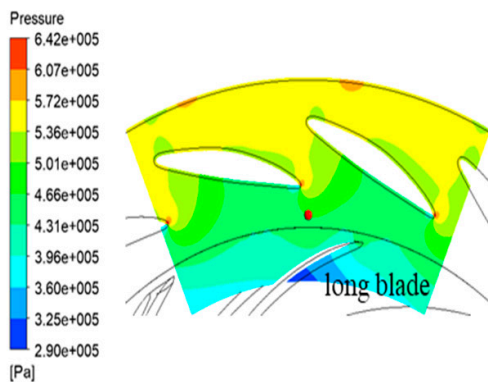

(e) $\mathrm{t}=0.02303 \mathrm{~s}$.
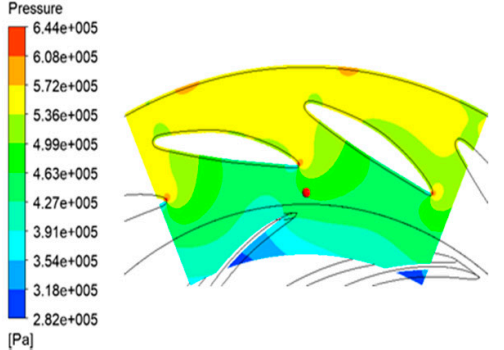

(c) $\mathrm{t}=0.01970 \mathrm{~s}$.
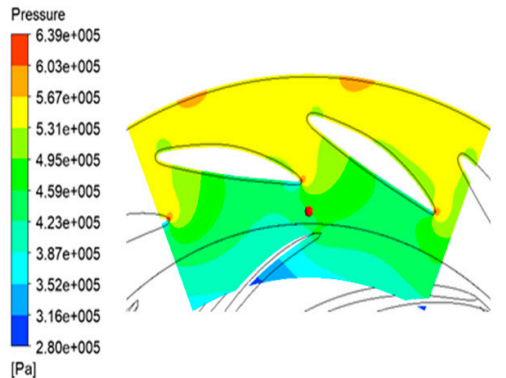

(f) $t=0.02455 \mathrm{~s}$.

Figure 14. Pressure distribution of GR001 at different times at $1.0 \mathrm{Q}_{\mathrm{d}}$.

In order to reflect the influence of the relative positions of the blades and stay guide vanes on pressure distribution in the bladeless region, Figure 14 shows the pressure distribution of GR001 at different times at $1.0 \mathrm{Q}_{\mathrm{d}}$, and a time domain diagram is also marked out, with the range from peak to trough of the pressure fluctuation. At $t=0.01758 \mathrm{~s}$, GR001 was close to the pressure surface of the short blade, and the pressure in the passage was relatively large, so the pressure fluctuation was at the peak at this moment. At $t=0.01909 \mathrm{~s}$, GR001 was close to the small pressure area near the head of the short blade, so the pressure fluctuation was negative at this moment. At $t=0.01970 \mathrm{~s}$, a short blade skimmed over GR001. The pressure rose, so the pressure fluctuation became positive at this moment. At $t=0.02152 \mathrm{~s}$, GR001 was far away from the short blade, and the influence of rotor-stator interference was weakened. Under the influence of the low pressure on the suction surface of the short blade, the pressure dropped and the pressure fluctuation became negative. At $t=0.02303 \mathrm{~s}$, GR001 was close to the pressure surface of the long blade, so the pressure fluctuation was at the secondary crest at this moment. At $t=0.02455 \mathrm{~s}$, GR001 was close to the small pressure area near the head of the long blade, so the pressure fluctuation was at a valley value at this moment. This indicates that rotation of the long and short blades will cause different pressure distributions; short blades have a more significant effect on the rise of pressure than long blades, while long blades have a more significant effect on the fall of pressure.

Figure 15 shows the circumferential distribution of rotor-stator interaction amplitudes at $1.0 \mathrm{Q}_{\mathrm{d}}$. It can be seen that the variation period of $5 \mathrm{f}_{n}$ was about $90^{\circ}$, which means that if the circumference area is divided into four quadrants, the distribution of this component in each quadrant is roughly the same. The variation period of $10 \mathrm{f}_{\mathrm{n}}$ and $20 \mathrm{f}_{\mathrm{n}}$ varied with a period of $22.5^{\circ}$, which was the same as the angle between each blade. It shows that the blade frequency and frequency doubling amplitude values were mainly affected by the different positions of the monitoring points relative to the adjacent guide vanes. In addition, the amplitude variation of the main frequency $10 \mathrm{f}_{\mathrm{n}}$ at the monitoring points GR037 GR039 was different from that at other points. Figure 15 shows the velocity distribution around GR001 GR003 and GR037 GR039 at 1.0 $Q_{d}$. It can be seen in Figure 16 that the high-speed flow field at the outlet of the blade impinged the guide vane, resulting in delamination on the trailing edge of 
the guide vane. The velocity direction was toward monitoring point GR002, so this monitoring point was greatly affected by the blade frequency. At monitoring points GR001 and GR003, at which the occurrence and attenuation of stay guide vane rotor-stator interaction occurred, respectively, the blade frequency amplitude was small. The variation trend of blade frequency amplitude at monitoring points GR037 GR039 was the same as that at monitoring points GR001 GR003. However, the upstream channel was near the tongue, so the insufficient overcurrent capacity led to a small velocity in the upstream, the runner's outflow was squeezed, and the velocity of the channel at the monitoring point increased. At monitoring point GR038, the velocity along the guide vane passage direction was relatively high while the upstream velocity was relatively low. The rotor-stator interaction effect generated after hitting the trailing edge of the guide vane was relatively small, resulting in almost no increase in the blade frequency amplitude at GR037 and GR038. Therefore, the blade frequency amplitude of GR038 and GR039 was obviously lower than that of the other channels at the same position.

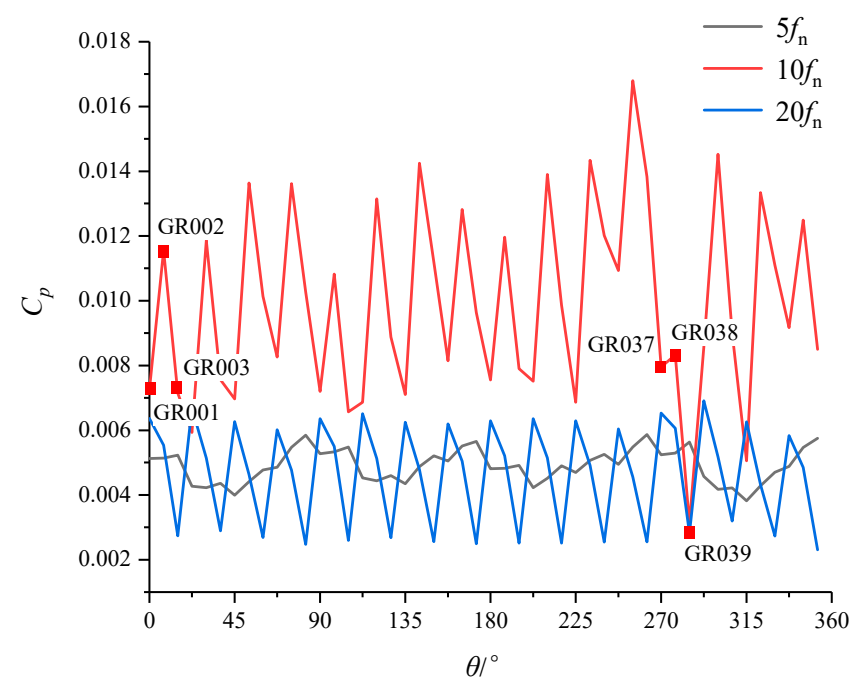

Figure 15. Circumferential distribution of rotor-stator interaction amplitudes at $1.0 \mathrm{Q}_{\mathrm{d}}$.

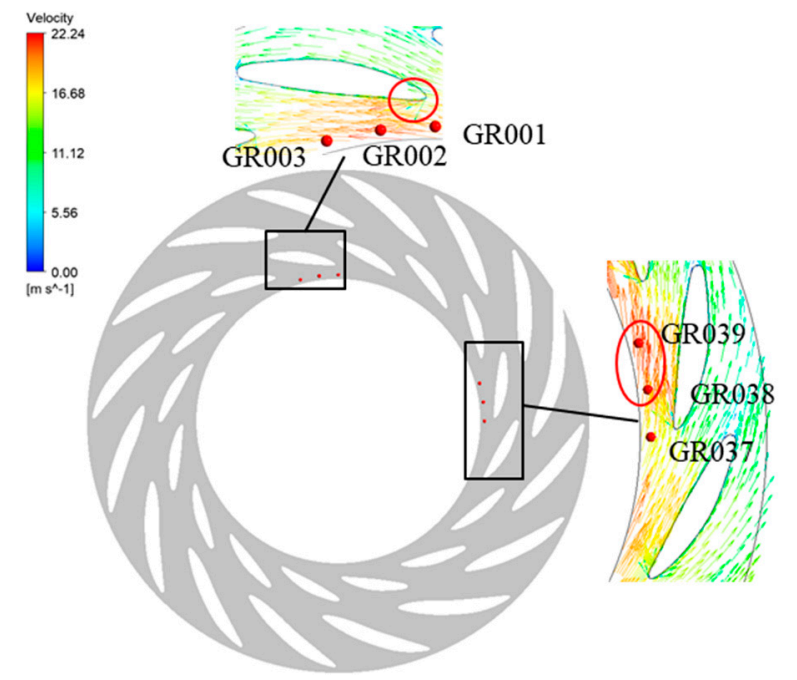

Figure 16. Velocity distribution around the monitoring points at $1.0 \mathrm{Q}_{\mathrm{d}}$.

As shown in Figures 17-19, the pressure fluctuation distributions at different vane heights under different flow rates were significantly different. The section near the roof was span 0.1 , span 0.5 was the middle section, and span 0.9 was the section near the bottom ring. At a low flow rate at $0.75 Q_{d}$, the pressure fluctuation intensity distribution at different guide vane heights had the most significant 
difference. Pressure fluctuation in span0.1 was small, and the strong fluctuation region was mainly concentrated below the trailing edge of the guide vane. The pressure fluctuation under the trailing edge of the guide vane at the middle height was significantly intensified, which affected the pressure fluctuation distribution in the bladeless region. The pressure fluctuation under the trailing edge of the active guide vane in span 0.9 weakened, but the pressure fluctuation intensity in the bladeless region near the outlet of the runner was greater. In addition, due to the strong flow separation near the trailing edge of the guide vane at a low flow rate at $0.75 \mathrm{Q}_{\mathrm{d}}$, the pressure fluctuation in the channel above the trailing edge of the guide vane was also obvious, especially near the bottom ring. With the increase of flow, the overcurrent capacity in the cascade channel increases, and the pressure fluctuation greatly reduces. The strong fluctuation region was mainly concentrated in the bladeless region near the runner's outlet, and the pressure fluctuation intensity near the bottom ring was relatively large. This indicates that the pressure fluctuation intensity at $0.75 \mathrm{Q}_{\mathrm{d}}$ is relatively large, and the flow separation at the trailing edge of the guide vane has a great influence on the pressure fluctuation in the bladeless region and the cascade channel. The pressure fluctuation intensity at $1.0 \mathrm{Q}_{\mathrm{d}}$ and $1.25 \mathrm{Q}_{\mathrm{d}}$ was relatively small, and the pressure fluctuation distributions in the bladeless region were mainly affected by the rotor-stator interaction at the outlet of the runner.

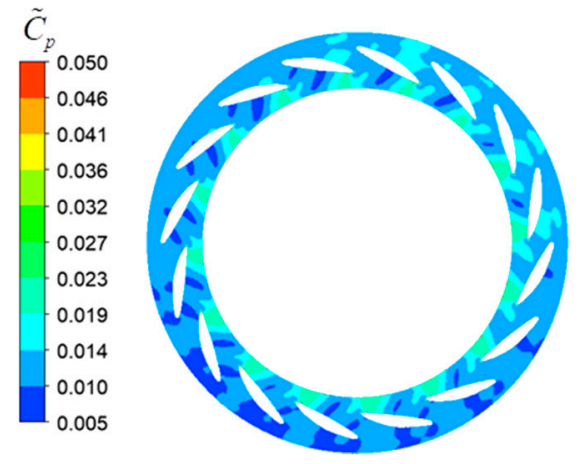

(a) span0.1.

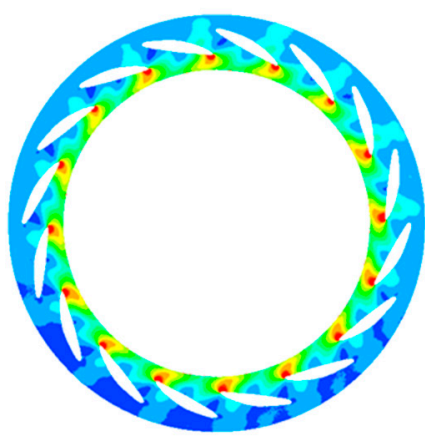

(b) span0.5.

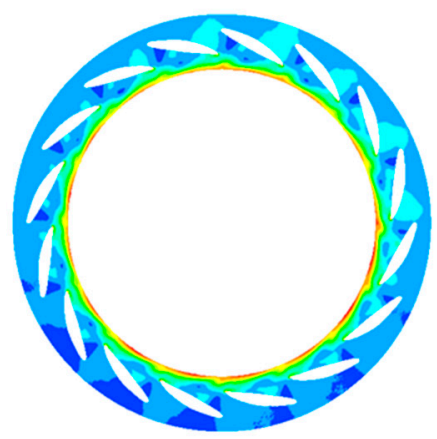

(c) span0.9.

Figure 17. Pressure fluctuation distributions at different vane heights at $0.75 Q_{d}$.

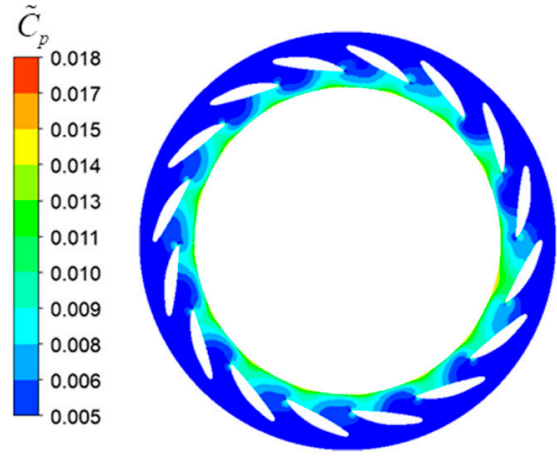

(a) span0.1.

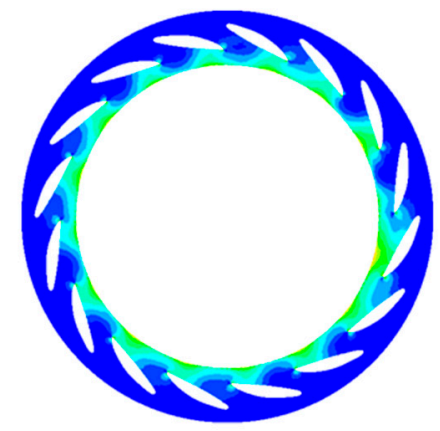

(b) span0.5.

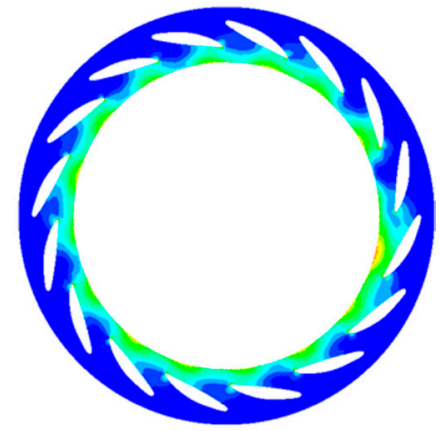

(c) span0.9.

Figure 18. Pressure fluctuation distributions at different vane heights at $1.0 \mathrm{Q}_{\mathrm{d}}$. 


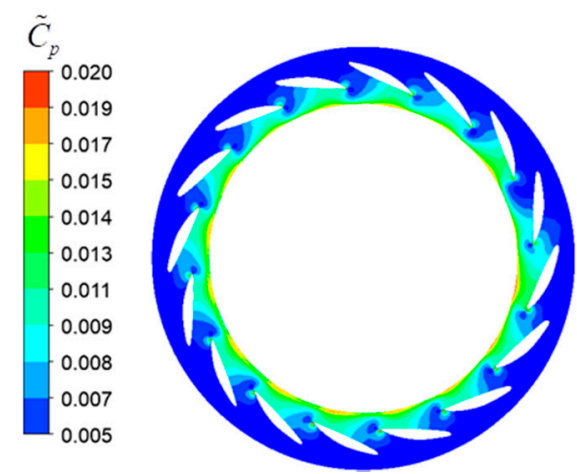

(a) span0.1.

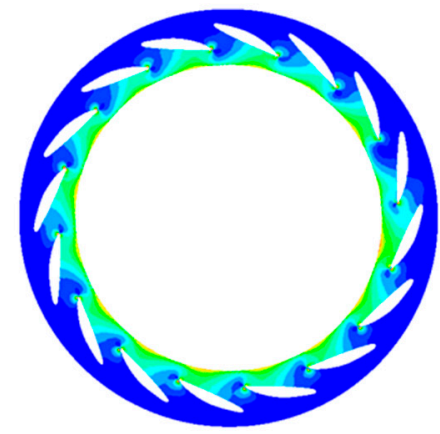

(b) span0.5.

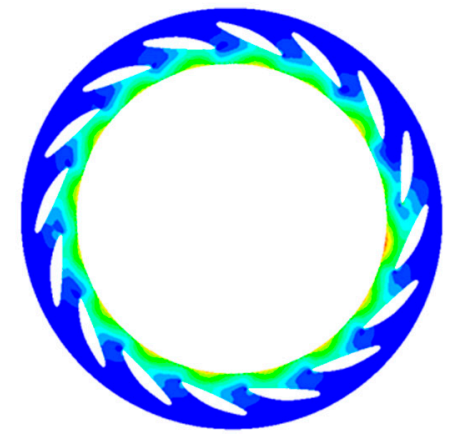

(c) span0.9.

Figure 19. Pressure fluctuation distributions at different vane heights at $1.25 \mathrm{Q}_{\mathrm{d}}$.

It can be seen from the above figures that the pressure fluctuation in the bladeless region at different vane heights has significant differences, and the high-amplitude fluctuation frequency caused by rotor-stator interaction is mainly $10 \mathrm{f}_{\mathrm{n}}$ and $20 \mathrm{f}_{\mathrm{n}}$. Figure 20 shows the circumferential distribution of $10 f_{n}$ and $20 f_{n}$ amplitudes at different vane heights and under different flow rates. As can be seen from the figure, the amplitude difference of $10 \mathrm{f}_{\mathrm{n}}$ at different vane heights was large, while the amplitude difference of $20 f_{n}$ was relatively insignificant. For $10 f_{n}$, at a small flow rate at $0.75 Q_{d}$, the amplitudes near the middle height and the bottom ring were relatively large, while the amplitude near the roof was significantly reduced, indicating that the rotor-stator interaction near the bottom ring was relatively strong. With the increase of the flow rate, the amplitude of the middle height gradually decreased, close to that found near the roof, and the amplitude near the bottom ring was relatively large, especially at $1.25 \mathrm{Q}_{\mathrm{d}}$. Except for the monitoring points near the trailing edge of the guide vanes, the amplitudes of $10 f_{n}$ at the other monitoring points were higher than $20 f_{n}$, indicating that the node-diameter number $k_{1}=-6$ dominated the rotor-stator interaction in the bladeless region.

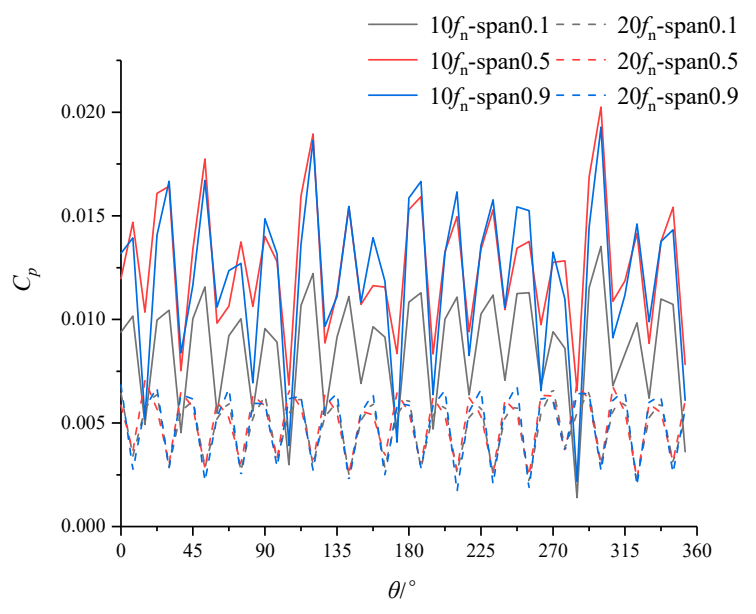

(a) $0.75 \mathrm{Qd}$.

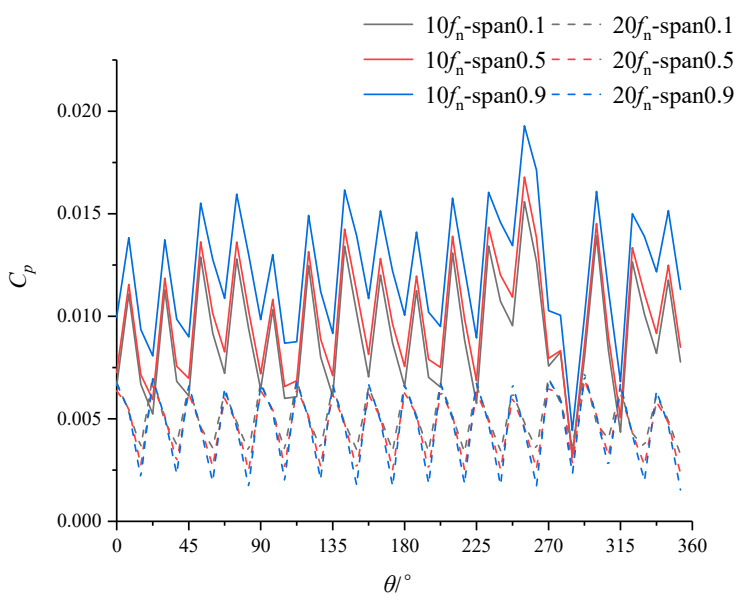

(b) $1.0 \mathrm{Q}_{\mathrm{d}}$.

Figure 20. Cont. 


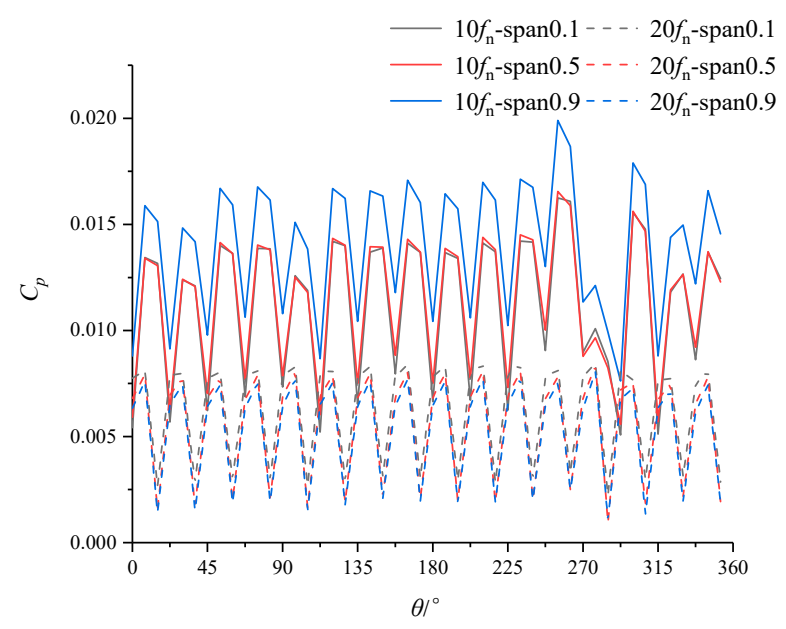

(c) $1.25 \mathrm{Q}_{\mathrm{d}}$.

Figure 20. Circumferential distribution of $10 f_{n}$ and $20 f_{n}$ amplitudes at different vane heights.

\subsection{Pressure Fluctuation Analysis of Volute}

Figure 21 shows the pressure fluctuation distributions at the mid-span of the volute in five rotation cycles under different flow rates. It can be seen that the flow rate had a significant influence on the intensity and distribution characteristics of pressure fluctuation in the volute. At a low flow rate at $0.75 Q_{d}$, the pressure fluctuation at the tongue was the most violent, and the fluctuation intensity of the pressure surface decreased gradually with the increase of the section area, forming the pressure fluctuation gradient, which reached the minimum value at the farthest distance from the tongue and then maintained stability at the outlet direction. The pressure fluctuation intensity at a rated flow rate at $1.0 \mathrm{Q}_{\mathrm{d}}$ was small and uniformly distributed, and there was no gradient change. As the flow rate increased, compared with other positions, the pressure fluctuation of the flow passage with a small section near the tongue increased significantly, but it was still about $10^{-1}$ times that of the same position with a small flow rate. This indicates that deviated conditions, especially low flow conditions, will lead to a significant increase in pressure fluctuation of the flow passage at a small cross-section near the tongue, which may seriously induce strong vibrations.

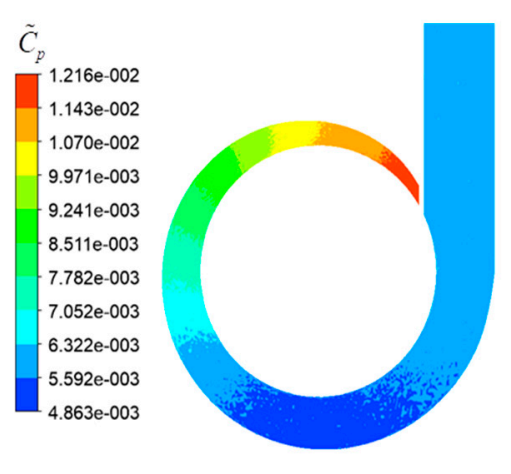

(a) 0.75 Qd.

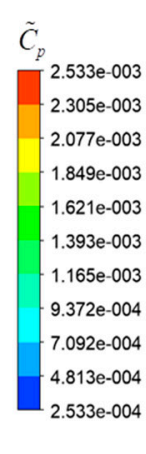

(b) $1.0 Q_{\mathrm{d}}$.
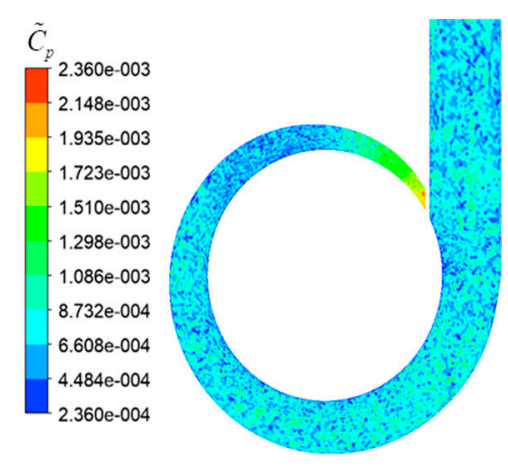

(c) $1.25 \mathrm{Qd}$.

Figure 21. Pressure fluctuation distributions at the mid-span of the volute.

In the pump working condition, as a downstream component, the unsteady pressure distribution of the volute is susceptible to the influence of rotor-stator interaction in the upstream, especially in the bladeless region. Figure 22 shows the distribution of $5 f_{n}$ and $10 f_{n}$ amplitudes for each monitoring point in the volute. As can be seen from the figure, the pressure fluctuation amplitude at the mid-span 
of the volute at a low flow rate at $0.75 Q_{d}$ was strong at $7.8 \mathrm{f}_{\mathrm{n}}$, and the spectral feature was nonlinear, which is consistent with the fluctuation characteristic in the bladeless region. Monitoring point SC01, which was near the tongue, had the maximum amplitude, while monitoring point SC08 far from the tongue had the minimum amplitude. With the increase of flow, the pressure fluctuation at the mid-span of the volute was mainly concentrated in the rotor-stator interaction frequencies of $5 \mathrm{f}_{\mathrm{n}}, 10 \mathrm{f}_{\mathrm{n}}$, $15 f_{n}$, and $20 f_{n}$, especially in the blade frequency of $10 f_{n}$ and the single-type blade passing frequency of $5 \mathrm{f}_{\mathrm{n}}$. In order to further analyze the distribution rules of these two frequencies at the mid-span of the volute, Figure 23 shows the distribution of $5 f_{n}$ and $10 f_{n}$ amplitudes in the volute at rated flow rates at $1.0 \mathrm{Q}_{\mathrm{d}}$ and $1.25 \mathrm{Q}_{\mathrm{d}}$. It can be seen that, for the blade frequency of $10 \mathrm{f}_{\mathrm{n}}$, the amplitude at $1.25 \mathrm{Q}_{\mathrm{d}}$ was higher than that at $1.0 \mathrm{Q}_{d}$, and the distribution of the two in the volute were similar. From SC01 to $\mathrm{SC} 05,10 \mathrm{f}_{\mathrm{n}}$ had a high attenuation rate, reached a minimum value at SC08-SC09 (far from the tongue), and was stable near the outlet of the volute. For a single-blade passing frequency of $5 f_{n}$, the amplitude of the blade from the small section to the large section gradually decreased at the rated flow rate at $1.0 \mathrm{Q}_{\mathrm{d}}$. While at the large flow rate at $1.25 \mathrm{Q}_{\mathrm{d}}$, the amplitude of SC01-SC06 at the small section near the tongue was less than the rated flow rate, but the amplitude of SC07-SC12 at the large section near the exit was significantly increased and higher than the rated flow rate. This indicates that the change of flow rate changes the propagation characteristic of $5 \mathrm{f}_{\mathrm{n}}$, thus changing the amplitude distribution rule corresponding to $5 \mathrm{f}_{\mathrm{n}}$. In general, the main frequency of pressure fluctuation in the small section of the volute flow passage was $10 f_{n}$, while in the big section it was $5 f_{n}$, indicating that the flow difference at the outlet of the splitter blades had a greater influence on the pressure fluctuation distribution than the blade passing interaction did.

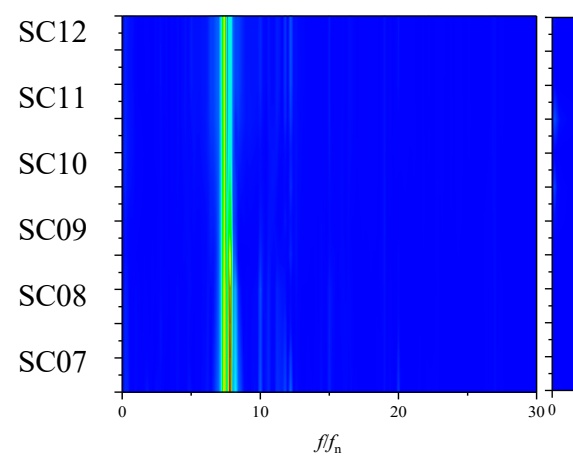

(a) $0.75 \mathrm{Qd}$.

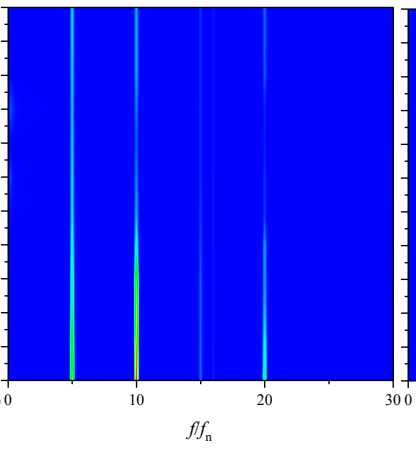

(b) $1.0 \mathrm{Qd}_{\mathrm{d}}$.

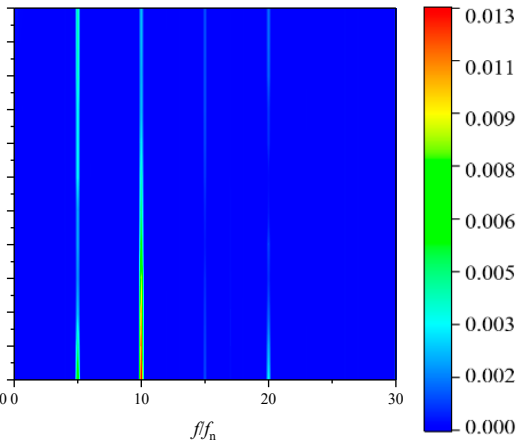

(c) $1.25 \mathrm{Qd}$.

Figure 22. Frequency domain of pressure fluctuation at the mid-span of the volute.

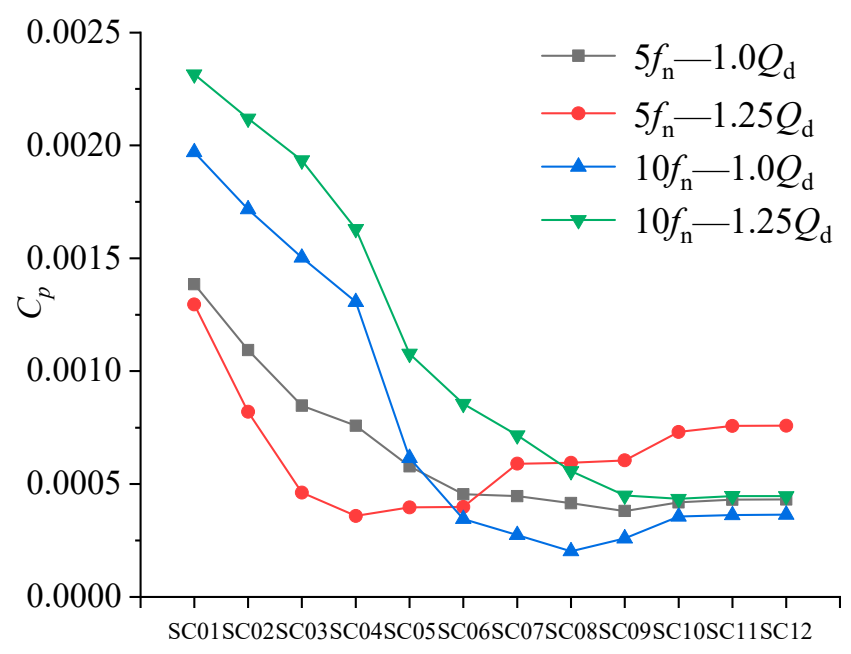

Figure 23. Distribution of $5 f_{n}$ and $10 f_{n}$ amplitudes in the volute. 


\subsection{Pressure Fluctuation Analysis of Draft Tube}

As an inflow component, the rotor-stator interaction and flow instability of the draft tube were mainly near the outlet of the draft tube. Figure 24 shows the distributions of the standard deviation coefficient of pressure fluctuation at the outlet of the draft tube under different flow rates within five rotation cycles. It can be seen that pressure fluctuation distributions under different flow rates at the outlet were similar. The pressure fluctuation intensity near the center was small and increased continuously along the radial direction. The pressure fluctuation intensity at the tube wall was about $10^{2}$ times that at the center. In addition, the fluctuation intensity was the highest at $0.75 \mathrm{Q}_{\mathrm{d}}$ and the lowest at $1.0 \mathrm{Q}_{\mathrm{d}}$, indicating that deviated working conditions, especially at a small flow rate, will aggravate the pressure fluctuation at the outlet of the draft tube, which damages the stable operation of the unit.
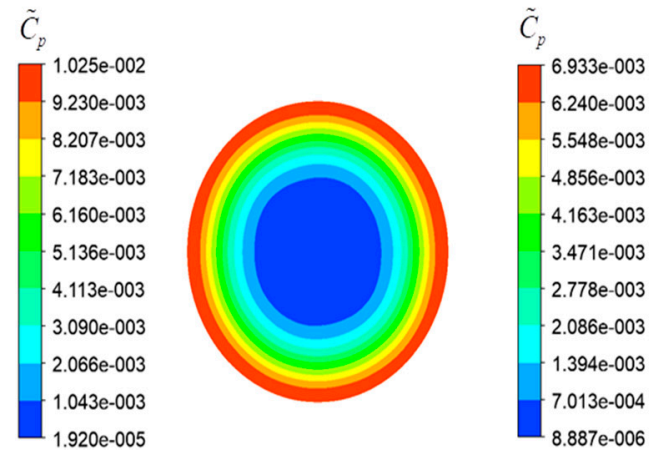

(a) $0.75 Q_{d}$.

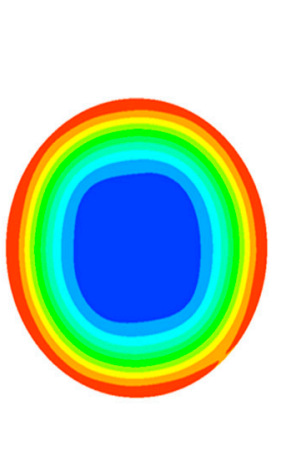

(b) $1.0 \mathrm{Qd}$.
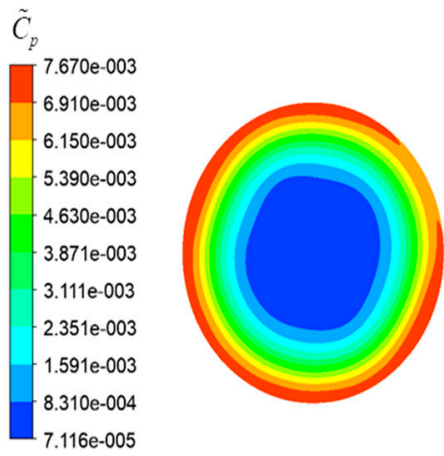

(c) $1.25 \mathrm{Qd}$.

Figure 24. Pressure fluctuation distributions at the outlet of the draft tube.

In order to further analyze the time and frequency domain characteristics of the pressure fluctuation of the draft tube under different flow rates, Figure 25 shows the time and frequency domain of pressure fluctuation at the outlet of the draft tube at monitoring points DT01, DT02, and DT03 within a rotational cycle. The results show that the pressure fluctuation distributions of DT01 and DT03 at the tube wall were similar. There are five peaks and valleys in a rotation period, which are affected by the rotor-stator interaction between the long blade and the outlet of the draft tube. In the time domain figures, the main frequency is $5 f_{n}$, and the secondary main frequency is $10 f_{n}$. The fluctuation amplitude under a rated flow rate at $1.0 Q_{d}$ was relatively small, while the negative amplitude under a small flow rate at $0.75 Q_{d}$ was relatively large, resulting in large pressure fluctuations under this working condition. In addition, by comparing the time domain figure of each flow rate, it can be found that the change of flow rate also has an obvious influence on the phase of pressure fluctuation. For the central monitoring point DT02, the time domain distribution showed an irregular periodic fluctuation, and the fluctuation amplitude under $1.25 \mathrm{Q}_{\mathrm{d}}$ was large, but the fluctuation amplitude was about two orders of magnitude lower than that of the monitoring point at the tube wall. In the frequency domain figures, it is shown that the main frequencies are axis-doubling frequencies, the main frequency is $5 f_{n}$, and the amplitude at $1.25 Q_{d}$ is relatively large, indicating that the influence of rotor-stator interaction between the runner and the draft tube on the middle position of the interface is relatively significant at a large flow rate. 


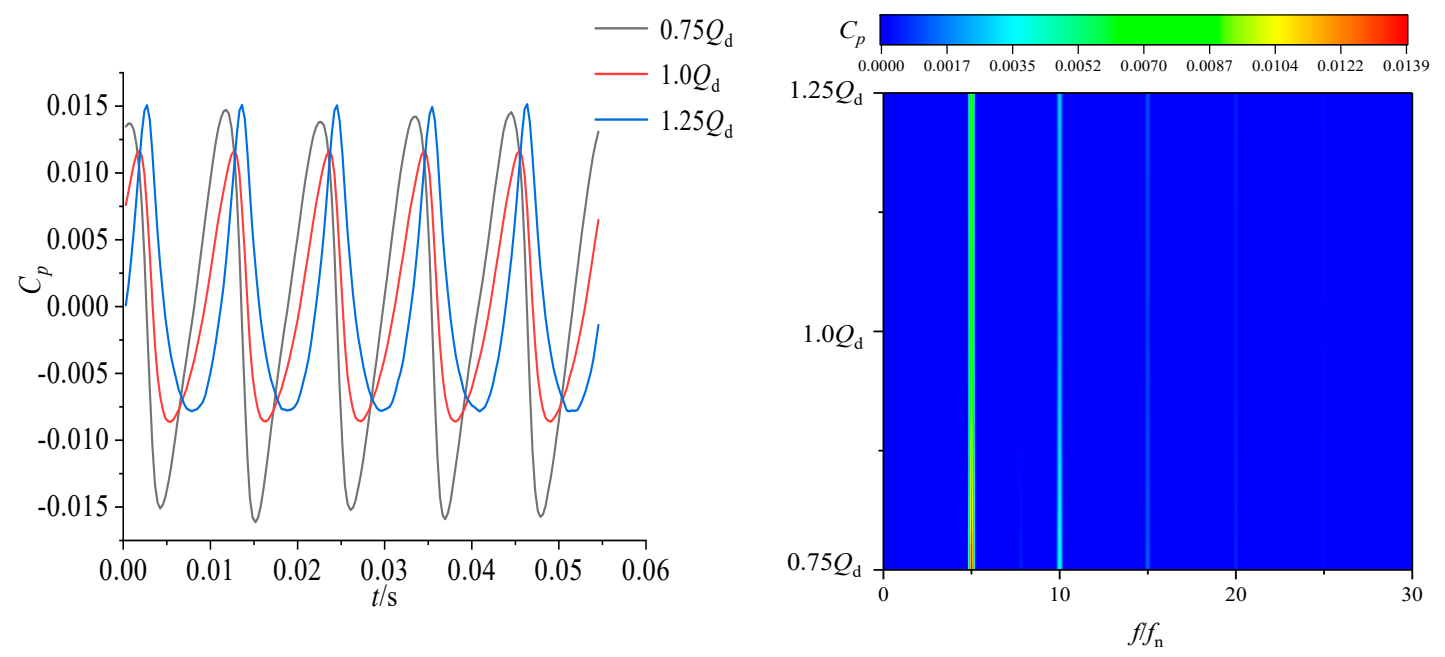

(a) Time domain of DT01.

(b) Frequency domain of DT01.
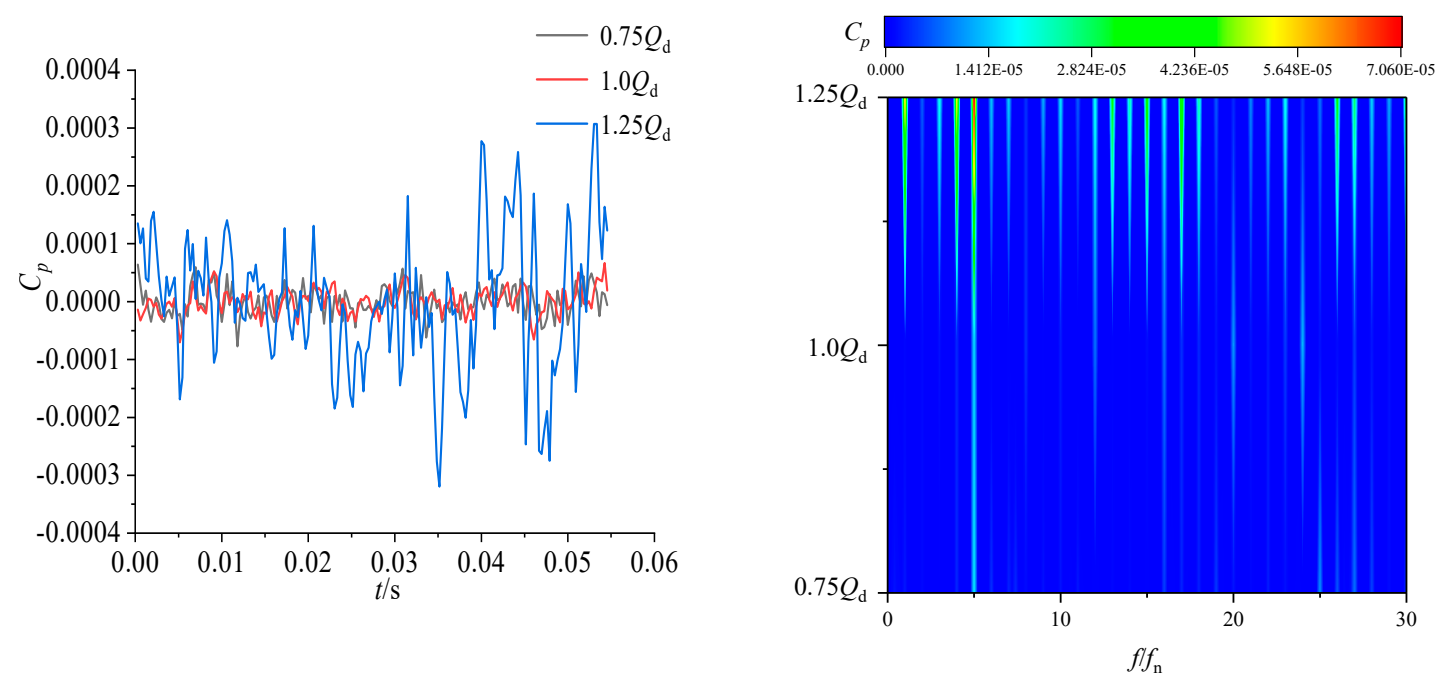

(c) Time domain of DT02.

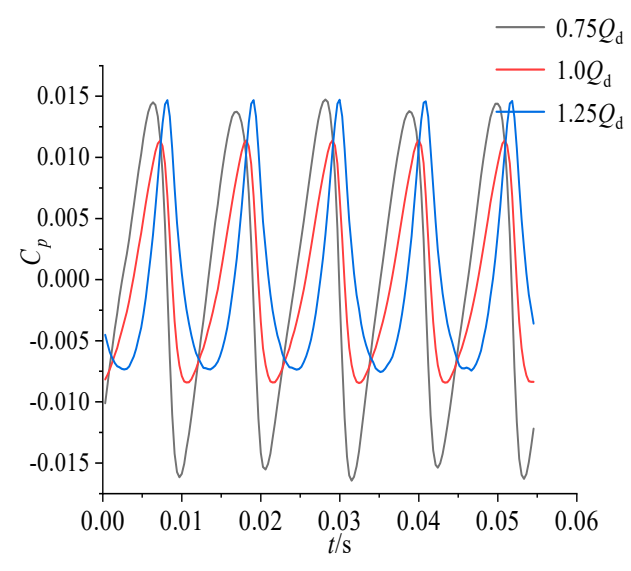

(e) Time domain of DT03.

(d) Frequency domain of DT02.

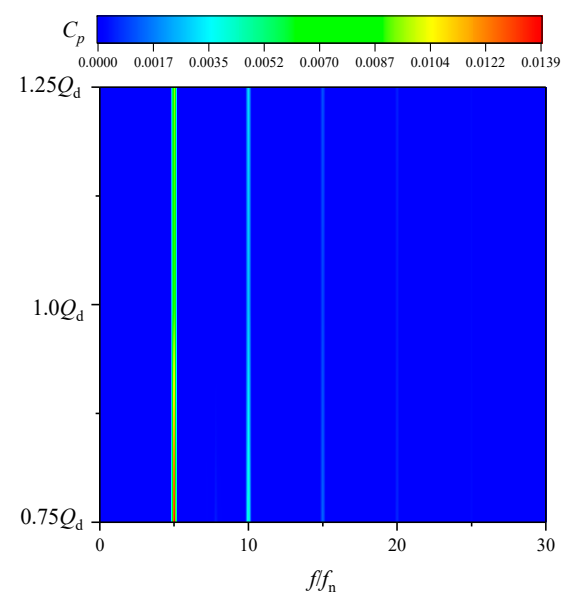

(f) Frequency domain of DT03.

Figure 25. Time and frequency domain of pressure fluctuation at the outlet of the draft tube. 
Figure 26 shows the frequency domain of pressure fluctuation of DT04-DT12 under different flow rates. The results show that the main frequencies of pressure fluctuation at these positions were axis-doubling frequencies, in which $10 \mathrm{f}_{\mathrm{n}}, 16 \mathrm{f}_{\mathrm{n}}$, and $20 \mathrm{f}_{\mathrm{n}}$ were caused by the upstream transmission of the rotor-stator interaction between the bladeless region and the runner. Overall, with the increase of the flow rate, the pressure fluctuation amplitude increased gradually from the inlet of the draft tube to the straight taper tube, and the amplitude at monitoring points DT04-DT09, which were near the elbow position and outer wall of the draft tube, was larger. Moreover, the amplitude of the outer wall of the tube was slightly larger than that of the inner wall, which was mainly due to the rotor-stator interaction between the runner and the draft tube, as well as the strong impact of the water flowing through the elbow tube on the outer wall.

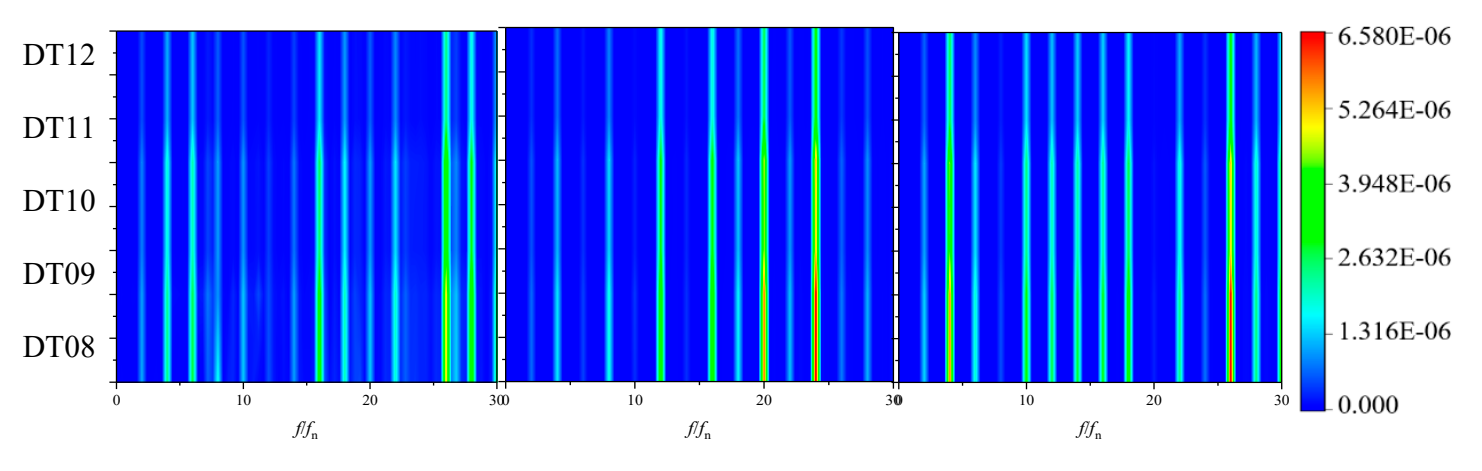

(a) $0.75 Q_{\mathrm{d}}$.

(b) $1.0 \mathrm{Qd}$.

(c) $1.25 \mathrm{Qd}$.

Figure 26. Frequency domain of pressure fluctuation of DT04-DT12.

\section{Conclusions}

The intensity and frequency distribution of pressure fluctuation under a low flow rate at $0.75 Q_{d}$, rated flow rate at $1.0 \mathrm{Q}_{\mathrm{d}}$, and high flow rate at $1.25 \mathrm{Q}_{\mathrm{d}}$ were analyzed. The influence of different flow rates on the rotor-stator interaction in the pump-turbine were analyzed in detail. The main results are as follows:

1. The pressure fluctuation analysis of the runner concludes that, under the influence of rotor-stator interaction, the fluctuation near the trailing edge pressure surface of the long and short blades was the most violent. At $0.75 \mathrm{Q}_{\mathrm{d}}$, compared with $1.0 \mathrm{Q}_{\mathrm{d}}$ and $1.25 \mathrm{Q}_{\mathrm{d}}$, flow instability led to a greater pressure fluctuation intensity which, to some extent, intensified the rotor-stator interaction at the blade's trailing edge, and the amplitude of the nonlinear frequency exceeded the rotor-stator interaction frequency.

2. The pressure fluctuation analysis of the bladeless region and double-row cascade concludes that, at $0.75 \mathrm{Q}_{\mathrm{d}}$, compared with $1.0 \mathrm{Q}_{\mathrm{d}}$ and $1.25 \mathrm{Q}_{\mathrm{d}}$, strong flow instability led to more nonlinear frequency components, and the flow rate would affect the energy distribution of rotor-stator interaction at different node-diameter numbers. From the bladeless region to the outlet of the stay vanes, the amplitude of the rotor-stator interaction frequency attenuated continuously, and the amplitude attenuation of each frequency was mainly concentrated in the area of the guide vanes. The amplitude attenuation rate of the stay vane region was small, indicating that the frequency passability of the guide vane region was low while the frequency passability of the stay vane region was high.

3. The pressure fluctuation analysis of the volute concludes that the flow rate had a significant impact on the pressure fluctuation intensity and distribution characteristics in the volute. The pressure fluctuation intensity at the tongue was the most violent at $0.75 \mathrm{Q}_{\mathrm{d}}$. The pressure surface fluctuation intensity gradually decreased along the direction of the increase in the section area, forming a pressure fluctuation gradient and strong nonlinear spectral characteristics. In contrast, the pressure fluctuation intensity at $1.0 \mathrm{Q}_{\mathrm{d}}$ and $1.25 \mathrm{Q}_{\mathrm{d}}$ was small and evenly distributed. In addition, the flow difference at the outlet of the long and short blades had great influence on the pressure fluctuation at the large section of the volute. 
4. The analysis of the pressure fluctuation of the draft tube concludes that the pressure fluctuation near the outlet wall of the draft tube was more violent under the influence of rotor-stator interaction, especially in the working condition of the small flow. The protruding frequencies of pressure fluctuation at the inlet of the liner tube to the straight taper tube were all axial multiples, the amplitude of pressure fluctuation near the outlet and the elbow tube was larger, and the amplitude of pressure fluctuation outside the tube wall was slightly larger than that inside the tube wall.

Author Contributions: Conceptualization, J.Z. and Y.X.; methodology, H.C. and H.S.; formal analysis, P.H.; resources, J.Z.; writing—original draft preparation, Y.X. and H.C.; writing—review and editing, H.S.; supervision, J.Z. and P.H. All authors have read and agreed to the published version of the manuscript.

Funding: This research was funded by the National Natural Science Foundation of China: 51809121 and 51779107, Natural Science Foundation of Jiangsu Province: BK20170548, Special Supported Project of China Postdoctoral Science Foundation: 2019T120394, and the National Key R\&D Program of China: 2017YFC0806604.

Conflicts of Interest: On behalf of all authors, the corresponding author states that there is no conflict of interest.

\section{References}

1. Li, D.Y.; Lin, S.; Wang, H.J.; Fu, W.W.; Chen, J.X.; Wei, X.Z.; Qin, D.Q. Influence of cavitation on hump characteristics in a pump-turbine model. IOP Conf. Ser. Earth Environ. Sci. 2019, 240, 072031. [CrossRef]

2. Agnalt, E.; Iliev, I.; Solemslie, B.W.; Dahlhaug, O.G. On the Rotor Stator Interaction Effects of Low Specific Speed Francis Turbines. Int. J. Rotat. Mach. 2019, 2019, 5375149. [CrossRef]

3. Ran, H.; Liu, Y.; Luo, X.; Shi, T.; Xu, Y.; Chen, Y.; Wang, D. Experimental comparison of two different positive slopes in one single pump turbine. Renew. Energy 2020, 154, 1218-1228. [CrossRef]

4. Tao, J.; Wang, H.; Liao, H.; Yu, S. Mechanical design and numerical simulation of digital-displacement radial piston pump for multi-megawatt wind turbine drivetrain. Renew. Energy 2019, 143, 995-1009. [CrossRef]

5. Khanjari, A.; Mahmoodi, E.; Ahmadi, M.H. Energy and exergy analyzing of a wind turbine in free stream and wind tunnel in CFD domain based on actuator disc technique. Renew. Energy 2020, 160, 231-249. [CrossRef]

6. Azad, S.; Lotfi, H.; Riasi, A. The effects of viscoelastic fluid on the cavitation inception and development within a centrifugal pump: An experimental study. Int. Commun. Heat Mass Transf. 2019, 107, 106-113. [CrossRef]

7. Fecser, N.; Sára, B.; Kuti, R. Examining Centrifugal Pump on Cavitation. Hidraulica 2019, 4, 6-12.

8. Ni, D.; Zhang, N.; Gao, B.; Li, Z.; Yang, M. Dynamic measurements on unsteady pressure pulsations and flow distributions in a nuclear reactor coolant pump. Energy 2020, 198, 117305. [CrossRef]

9. Keller, J.; Blanco, E.; Barrio, R.; Parrondo, J. PIV measurements of the unsteady flow structures in a volute centrifugal pump at a high flow rate. Exp. Fluids 2014, 55, 1820. [CrossRef]

10. Zhang, F.; Appiah, D.; Hong, F.; Zhang, J.; Yuan, S.; Adu-Poku, K.A.; Wei, X. Energy loss evaluation in a side channel pump under different wrapping angles using entropy production method. Int. Commun. Heat Mass Transf. 2020, 113, 104526. [CrossRef]

11. Wang, Y.; Zhang, F.; Yuan, S.; Chen, K.; Wei, X.; Appiah, D. Effect of URANS and Hybrid RANS-LES Turbulence Models on Unsteady Turbulent Flows Inside a Side Channel Pump. ASME J. Fluids Eng. 2020, 142, 061503. [CrossRef]

12. Jia, X.Q.; Zhu, Z.C.; Yu, X.L.; Zhang, Y.L. Internal unsteady flow characteristics of centrifugal pump based on entropy generation rate and vibration energy. Proc. Inst. Mech. Eng. 2019, 233, 456-473. [CrossRef]

13. Rodriguez, C.G.; Egusquiza, E.; Santos, I.F. Frequencies in the Vibration Induced by the Rotor Stator Interaction in a Centrifugal Pump Turbine. J. Fluids Eng. 2007, 129, 1428-1435. [CrossRef]

14. Gao, Z.; Zhu, W.; Lu, L.; Deng, J.; Zhang, J.; Wuang, F. Numerical and Experimental Study of Unsteady Flow in a Large Centrifugal Pump With Stay Vanes. J. Fluids Eng. 2014, 136, 071101. [CrossRef]

15. Botero, F.; Hasmatuchi, V.; Roth, S.; Farhat, M. Non-intrusive detection of rotating stall in pump-turbines. Mech. Syst. Signal Process. 2014, 48, 162-173. [CrossRef]

16. Stuermer, A.W.; Akkermans, R.A. Validation of Aerodynamic and Aeroacoustic Simulations of Contra-Rotating Open Rotors at Low-Speed Flight Conditions. In Proceedings of the 32nd AIAA Applied Aerodynamics Conference, Atlanta, GA, USA, 16-20 June 2014. 
17. Stuermer, A.; Yin, J.; Akkermans, R. Progress in aerodynamic and aeroacoustic integration of CROR propulsion systems. Aeronaut. J. 2014, 118, 1137-1158. [CrossRef]

18. Akkermans, R.A.D.; Stuermer, A.; Delfs, J.W. Active Flow Control for Interaction Noise Reduction of Contra-Rotating Open Rotors. AIAA J. 2016, 54, 1413-1423. [CrossRef]

19. Tyler, J.M.; Sofrin, T.G. Axial Flow Compressor Noise Studies. SAE Tech. Pap. Ser. 1962, 309-332. [CrossRef]

20. Yonezawa, K.; Toyahara, S.; Motoki, S.; Tanaka, H.; Doerfler, P.; Tsujimoto, Y. Phase Resonance in Centrifugal Fluid Machinery-A Comparison between Pump Mode and Turbine Mode Operations and a Discussion of Mechanisms of Flow Rate Fluctuation through a Stator. Int. J. Fluid Mach. Syst. 2014, 7, 42-53. [CrossRef]

21. Shigemitsu, T.; Fukutomi, J.; Wada, T.; Shinohara, H. Performance analysis of mini centrifugal pump with splitter blades. J. Therm. Sci. 2013, 22, 573-579. [CrossRef]

22. Cavazzini, G.; Pavesi, G.; Santolin, A.; Ardizzon, G.; Lorenzi, R. Using splitter blades to improve suction performance of centrifugal impeller pumps. Proc. Inst. Mech. Eng. 2015, 229, 309-323. [CrossRef]

23. Iwadachi, A.; Tani, K.; Aguro, K. The design of adjustable-speed pump-turbine modified from existing constant-speed on Okutataragi Power Station. In Proceedings of the 19th International Conference on Electrical Machines and Systems (ICEMS), Chiba, Japan, 13-16 November 2016.

24. Kubota, Y.; Suzuki, T.; Tomita, H.; Nagafugi, T.; Okamura, C. Vibration of Rotating Bladed Disc Excited by Stationary Distributed Forces. Bull. JSME 1983, 26, 1952-1957. [CrossRef]

25. Tanaka, H. Vibration Behavior and Dynamic Stress of Runners of Very High Head Reversible Pump-turbines. Int. J. Fluid Mach. Syst. 2011, 4, 289-306. [CrossRef]

(C) 2020 by the authors. Licensee MDPI, Basel, Switzerland. This article is an open access article distributed under the terms and conditions of the Creative Commons Attribution (CC BY) license (http://creativecommons.org/licenses/by/4.0/). 